\title{
Beyond the MSSM Higgs bosons at the Tevatron and the LHC
}

\author{
Carena, M ; Ponton, E ; Zurita, J
}

\begin{abstract}
We study extensions of the minimal supersymmetric standard model (MSSM) with new degrees of freedom that couple sizably to the MSSM Higgs sector and lie in the TeV range. After integrating out the physics at the $\mathrm{TeV}$ scale, the resulting Higgs spectrum can significantly differ from typical supersymmetric scenarios, thereby providing a window beyond the MSSM (BMSSM). Taking into account current LEP and Tevatron constraints, we perform an in-depth analysis of the Higgs collider phenomenology and explore distinctive characteristics of our scenario with respect to both the standard model and the MSSM. We propose benchmark scenarios to illustrate specific features of BMSSM Higgs searches at the Tevatron and the LHC.
\end{abstract}

DOI: https://doi.org/10.1103/PhysRevD.82.055025

Other titles: BMSSM Higgs Bosons at the Tevatron and the LHC

Posted at the Zurich Open Repository and Archive, University of Zurich ZORA URL: https://doi.org/10.5167/uzh-41876

Journal Article

Accepted Version

Originally published at:

Carena, M; Ponton, E; Zurita, J (2010). Beyond the MSSM Higgs bosons at the Tevatron and the LHC. Physical Review D, 82(5):055025.

DOI: https://doi.org/10.1103/PhysRevD.82.055025 


\title{
BMSSM Higgs Bosons at the Tevatron and the LHC
}

\author{
Marcela Carena \\ Theoretical Physics Department, Fermilab, Batavia, Illinois 60510, USA and \\ Enrico Fermi Institute, University of Chicago, 5640 Ellis Avenue, Chicago, Illinois 60637, USA \\ Eduardo Pontón \\ Department of Physics, Columbia University, 538 W. 120th St., New York, New York 10027, USA \\ José Zurita \\ Institut für Theoretische Physik, Universität Zürich, \\ Winterthurerstrasse 190, CH-8057 Zürich, Switzerland.
}

\begin{abstract}
We study extensions of the minimal supersymmetric standard model (MSSM) with new degrees of freedom that couple sizably to the MSSM Higgs sector and lie in the TeV range. After integrating out the physics at the $\mathrm{TeV}$ scale, the resulting Higgs spectrum can significantly differ from typical supersymmetric scenarios, thereby providing a window beyond the MSSM (BMSSM). Taking into account current LEP and Tevatron constraints, we perform an in-depth analysis of the Higgs collider phenomenology and explore distinctive characteristics of our scenario with respect to both the standard model and the MSSM. We propose benchmark scenarios to illustrate specific features of BMSSM Higgs searches at the Tevatron and the LHC.
\end{abstract}

\section{INTRODUCTION}

There has been a recent surge of interest in extensions of the minimal supersymmetric standard model (MSSM) by higher-dimension operators [1-11]. These can have an important impact on the Higgs sector, alleviating in particular the tension present in the MSSM that results from the LEP Higgs bounds. Such effective field theory (EFT) studies allow a model-independent description of a large class of extensions of the MSSM, and permit one to quantify the sense in which the Higgs sector can serve as a window beyond the MSSM (BMSSM).

This point of view was clearly put forward in Ref. [3], where it was emphasized that at leading order in $1 / M-$ where $M$ is the scale of the physics that is integrated outthe MSSM is extended by only two parameters. The surprisingly large effects of such higher-dimension operators can be understood from the fact that the MSSM Higgs potential is rather restricted at tree-level. The nonrenormalizable operators in the superpotential induce renormalizable (quartic) operators in the Higgs potential that are not present in the MSSM limit (at tree-level), so that in spite of the fact that their coefficients are "small" -of order $\mu / M$ - they correspond to qualitatively new effects. In fact, the operators thus induced can easily "destabilize" the MSSM-like minimum and lead to new minima that exist only as a direct result of the higher-dimension operators (i.e. the heavy physics). It was emphasized in [7] that such minima can be phenomenologically viable, can be studied within the EFT framework, and can explain the distinct properties induced by the heavy physics on the Higgs sector.

If the BMSSM physics is sufficiently heavy, the leading order analysis at order $1 / M$ can suffice. However, it is perfectly possible that $M$ is not too far from the electroweak (EW) scale, and that nevertheless the heavy physics may not be easy to see directly at the LHC, even if it is within its kinematic reach (e.g. heavy singlets that couple only through the Higgs sector). In such cases, the EFT approach is still useful to describe the properties of the MSSM Higgs sector. It turns out that the effects of order $1 / M^{2}$ are more important than naively expected. This observation also finds a simple explanation in the structure of the MSSM tree-level Higgs potential [8] together with the smallness of the MSSM tree-level quartic couplings (the root cause for a Higgs state lighter than the $Z$ mass in the MSSM at tree-level). The crucial point is that the leading order contributions to a subset of the quartic Higgs operators, from the heavy physics, first enter at order $1 / M^{2}$. Thus, these end up correcting a coefficient of order $g^{2}$ instead of a $1 / M$ effect, and can give a relevant correction even if the expansion parameter is relatively small. Nevertheless, it is important to appreciate that the fact that the first two orders in the expansion in $1 / M$ can even result in comparable contributions to the Higgs masses, in no way implies a breakdown of the EFT.

In Ref. [8] a detailed study of the consequences for the Higgs masses and couplings up to order $1 / M^{2}$ was given, and a selected number of phenomenological observations were already made, such as: enhanced gluon fusion production cross sections in a large number of cases, and the presence of "exotic" decay modes with more than one Higgs boson in the decay chain. In this work we analyze the constraints from LEP and the Tevatron on the neutral Higgs bosons, as well as the charged Higgs bounds from LEP [? ]. We also expand on the associated collider phenomenology, emphasizing the type of signals that can be expected at both the Tevatron and the LHC. We point out that due to the large corrections to the Higgs masses (especially to the CP-even Higgs bosons) the production and decay patterns can be markedly different from those in the MSSM. Examples include mod- 
els where both CP even Higgs bosons have significant branching fractions into gauge bosons, thus giving rise to spectacular signals such as two clearly defined peaks in the di-lepton invariant mass distribution. In addition, we observe new decay chains that allow for production of the "nonstandard" Higgs bosons without large $\tan \beta$ enhancements. It is possible that the full two-Higgsdoublet-model (2HDM) content can be mapped in detail, thus providing a clear and definite signal for physics beyond the standard model, and a rather detailed understanding of the mechanism of electroweak symmetry breaking (EWSB). If, in addition, relatively light superparticle signals are observed, as might be expected in these scenarios, a clear case for BMSSM physics could be established. Apart from the collider phenomenology induced indirectly by the heavy physics, higher-dimension operators have also been studied in the context of dark matter [12-14], cosmology [15] and EW baryogenesis [16$20]$, and it may be interesting to further explore the connections with collider physics.

This paper is organized as follows. In Sec. II, we summarize the most relevant aspects of the models under study. In Sec. III we discuss the modifications of the Higgs spectrum, which are the dominant factor in determining the Higgs collider phenomenology. In Sec. IV we discuss in detail the range of signatures uncovered by our survey, separating the analysis into the low and large $\tan \beta$ regimes. We conclude in Sec. V.

\section{EXTENDED SUSY HIGGS SECTORS AT A GLANCE}

As already mentioned, when considering BMSSM scenarios where the non-MSSM degrees of freedom have masses parametrically larger than the weak scale, an EFT approach is very useful. The fact that at leading order only two parameters are added to those in the MSSM makes this a rather economic extension [3], that nevertheless can significantly change the MSSM Higgs phenomenology. However, the same reason that makes these $1 / M$ suppressed effects rather important also implies that the next order in the $1 / M$ expansion can be phenomenologically relevant, without implying a breakdown of the EFT [8]. At order $1 / M^{2}$ there are several SUSY-preserving and SUSY-violating operators in the Kähler potential, the most important of which, in relation to the Higgs phenomenology, were listed in Ref. [8]. We refer the reader to this reference for the detailed form of such operators and how they affect the expressions for the Higgs masses and couplings. Here we restrict ourselves to a few general remarks that summarize the most relevant features for the present study (full details were given in the above reference).

First, it has to be pointed out that the higherdimension operators to order $1 / M^{2}$ can be easily generated from UV completions that include a combination of Higgs singlets, $S U(2)$ Higgs triplets, heavy W primes and $\mathrm{Z}$ primes. As argued in [8] the upshot is that the coefficients of the higher-dimension operators, from a lowenergy point of view, can be chosen in an uncorrelated manner. Although the EFT description to order $1 / M^{2}$ introduces a large number of parameters, which makes the framework more involved compared to the truncation at order $1 / M$, one should notice that this same feature gives additional handles to infer properties of the heavy sector from the properties of the low-energy degrees of freedom. In any case, since our goal is to survey the collider signal possibilities in a model-independent way (in a supersymmetric framework), we focus on a low-energy study based on the EFT at order $1 / M^{2}$, as described in [8]. [? ]

A random scan over parameter space was performed, and a set of points satisfying several constraints was selected. The set of points in this study satisfy:

- All the dimensionless coefficients parametrizing the higher-dimension operators are taken to be at most of order one, i.e. it is assumed that the heavy physics at $M$ is weakly coupled.

- Global minimum: since the scalar potential can present several minima, we make sure that the vacuum under study is the global one (at least within the EFT). We also check that there are no charge/color breaking minima, and for simplicity we restrict to the $\mathrm{CP}$ conserving case (checking that the global minimum does not break CP spontaneously).

- Robustness: there are no accidental cancellations that can render (not computed) higher orders in the $1 / M$ expansion more important than expected.

- "Light" SUSY spectrum: given that generically, and unlike in the MSSM, these models satisfy the LEP bounds on the Higgs mass at tree-level, there is no need for large radiative corrections. Naturalness suggests that in these models the SUSY spectrum would be expected to be light (in the few hundred $\mathrm{GeV}$ range, consistent with direct bounds).

- Agreement with EW precision constraints, in particular in regards to the Peskin-Takeuchi $T$ parameter [21]. These arise from three sources: a subset of the higher-dimension operators (as generated, for instance, by Higgs triplets), the details of the MSSM Higgs spectrum, and potential custodially-violating mass splittings in the sparticle spectrum. We emphasize that mild cancellations allow for higher-dimension operator effects that can have a non-negligible impact on the Higgs collider phenomenology.

All of the above constraints were described in detail in [8]. In addition, we impose the current collider bounds from LEP and the Tevatron using the code HiggsBounds v1.2.0 [22, 23]. [? ] To this we add the LEP bounds on 
charged Higgs production [24], and the newest combined result from the Tevatron in the WW channel [25], and in the inclusive tau search [26], that are not included in the currently available version of this code. We use HiggsBounds in the "effective coupling" mode, which requires effective couplings defined by

$$
g_{\phi X}^{2}=\frac{\Gamma(\phi \rightarrow X)}{\Gamma_{S M}(\phi \rightarrow X)},
$$

where $\phi=h, H, A$ is any of the neutral Higgs states, $\Gamma(\phi \rightarrow X)$ is the partial width in our model into any of the final states $X=s \bar{s}, c \bar{c}, b \bar{b}, \tau \bar{\tau}, W W, Z Z, \gamma \gamma$ or $g g$ (when applicable), and $\Gamma_{S M}(\phi \rightarrow X)$ is the partial width for a SM Higgs of the corresponding mass. Together with the total widths in our model (and in the SM), these effective couplings encode the information about branching fractions into these decay channels in our model.

We have implemented our tree-level expressions for the spectrum and Higgs couplings in HDECAY v3.4 [27]. This allows us to compute the Higgs partial decay widths, taking into account the QCD radiative corrections, that are known to be sizable (for a review, see [28]). In addition, we include the radiative corrections derived from the 1-loop RG improved effective potential due to supersymmetric particles [29], and the SUSY QCD/EW corrections to the Yukawa couplings [30, 31]. Loop contributions from the heavy physics that has been integrated out are suppressed by both a loop factor and by powers of $M$, hence they are expected to be negligible.

In all the plots that follow, we have fixed the following dimensionful parameters: $M=1 \mathrm{TeV}$, $\mu=m_{S}=200 \mathrm{GeV},[?]$ and for simplicity, we use a common value $M_{S U S Y}=300 \mathrm{GeV}$ and $A_{t}=A_{b}=0$ in the MSSM sparticle sector.[? ] The light superparticle spectrum implies that the loop contributions to the Higgs masses are modest, while the loop contributions to the Higgs couplings are more important and sensitive to the details of this spectrum [30-32]. The above choice of $M_{S U S Y}=300 \mathrm{GeV}$ is simply meant to illustrate the possible loop effects arising from relatively light superparticles. In particular, one can expect the first two generation squarks to be somewhat heavier to satisfy direct collider bounds [33, 34] or the sleptons could be somewhat lighter, without changing our generic conclusions regarding the Higgs collider phenomenology. Note also that the neutralino/chargino sector depends on parameters not affecting the Higgs sector directly, and in particular that we do not impose constraints from dark matter (in this work, we remain agnostic as to the identity of the DM candidate, but see [35]). We have also not imposed indirect constraints, such as those arising from $b \rightarrow s \gamma$, $B_{s} \rightarrow \mu^{+} \mu^{-}$and $g_{\mu}-2$, that have the potential to put important restrictions, but depend on the flavor structure of the soft SUSY-breaking parameters.

We consider two representative values of $\tan \beta: \tan \beta=$ 2 and $\tan \beta=20$. The $\mathrm{CP}$-odd mass was varied in the range $20-400 \mathrm{GeV}$. The upper bound is taken to ensure a proper separation between the light and heavy scales, as required by the EFT analysis. The very low mass range is expected to be severely constrained, but we defer the study of such region to future work. We turn next to a detailed description of the most important physical characteristics of the set of models in the scan, starting with the Higgs spectrum.

\section{MASSES OF LOW-ENERGY HIGGS BOSONS}

In this section we study the spectra of these models, analyzing the modifications with respect to the MSSM. Compared to the results already presented in [8], we include the 1-loop supersymmetric corrections to the Higgs quartic couplings as given in [29] (a minor effect for the relatively low SUSY spectrum we have in mind), as well as the constraints coming from collider data (LEP and Tevatron).

In Fig. 1 we show the mass of the lightest CP-even Higgs $(h)$ as a function of $m_{A}$, for both $\tan \beta=2$ (left panel) and $\tan \beta=20$ (right panel). The green points represent models ruled out by LEP, while the magenta ones are excluded by current data from CDF and D0. We divide the remaining allowed models into two subsets. First, one has those models that will be probed at the Tevatron at $95 \%$ C.L (red points), assuming $10 \mathrm{fb}^{-1}$ per experiment and $50 \%$ efficiency improvements [36] (see [37] for detailed projections in the MSSM context). These comprise the future reach of two search channels: $h / H \rightarrow b \bar{b}$ with the Higgs being produced in association with electroweak gauge bosons, and $g g \rightarrow h / H \rightarrow W^{+} W^{-}$. Second, the blue points are those that will be out of the Tevatron reach under the previous assumptions. For reference, we also show the MSSM (dashed) curve, assuming the same light SUSY spectrum. This color code will be employed in all the plots.

The corrections to $m_{h}$ due to the new physics are most important in the low $\tan \beta$ regime. Nevertheless, it is clear that they can also be relevant at large $\tan \beta$. The higher-dimension operators affect $m_{h}$ in such a way that it can easily be above the MSSM value. In the left plot, where $\tan \beta=2$, all the points lie above the MSSM curve; $m_{h}$ can reach values as high as $250 \mathrm{GeV}$.

Moreover, the left panel of Fig. 1 shows in a clear way how the Tevatron probes these models. For high enough values of $m_{A}$ one distinguishes mostly uniformly single colored horizontal stripes. The magenta one, where $m_{h} \sim$ $160-170 \mathrm{GeV}$, corresponds to $h$ being excluded by the current Tevatron search in the $W W$ channel [25]. Note that this range is slightly larger than the SM one (162$166 \mathrm{GeV})$. This is due to the fact that, in our models, the gluon fusion cross section can be mildly enhanced with respect to the SM one. By the same token, one understands the presence of a few red points within the magenta stripe as those corresponding to models whose gluon fusion cross section is below the SM value. The two red stripes $\left(m_{h}\right.$ in the ranges $140-160 \mathrm{GeV}$ and 

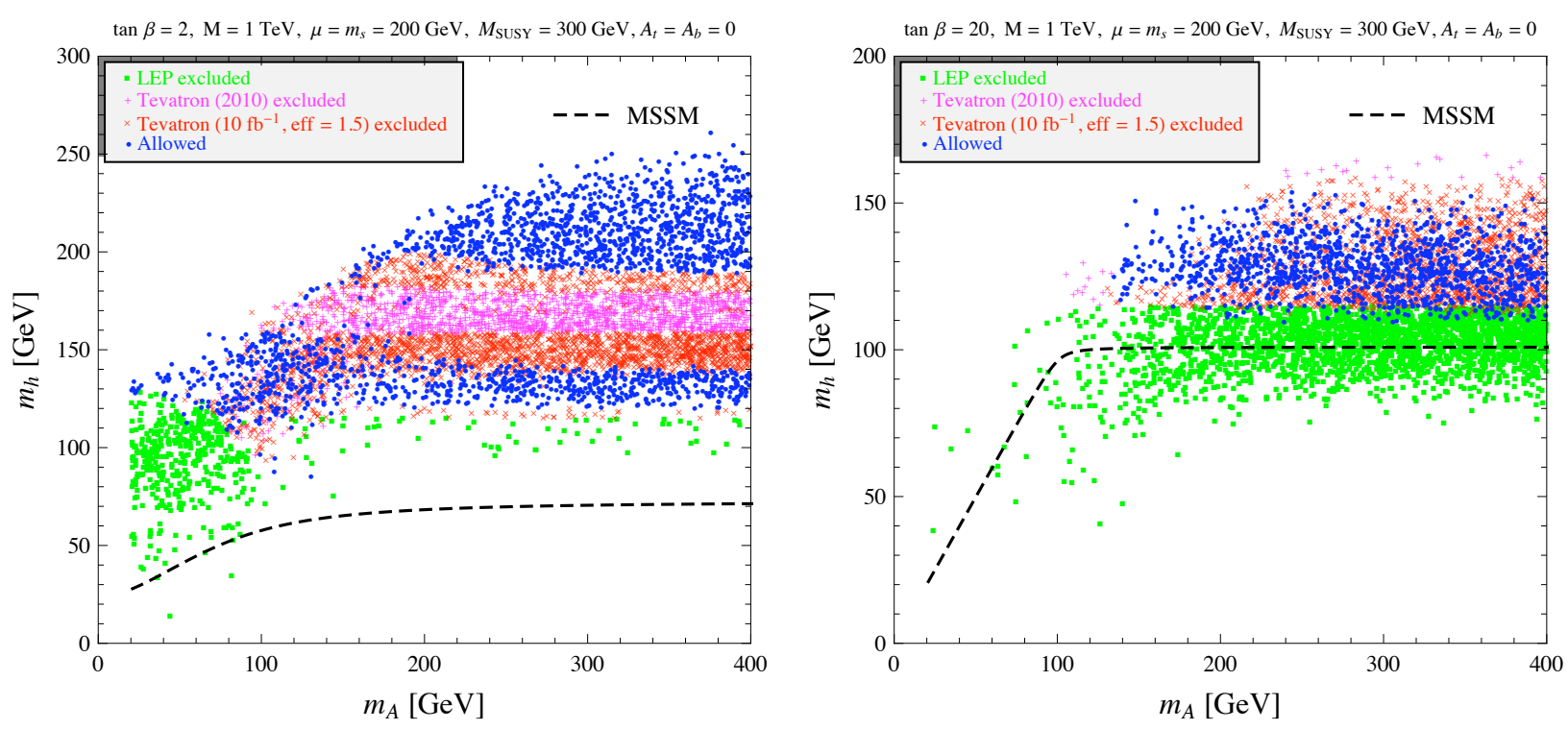

FIG. 1. Lightest CP-even Higgs boson mass as a function of $m_{A}$, for $\tan \beta=2$ (left panel) and $\tan \beta=20$ (right panel). We show the points excluded by LEP (green), excluded by current Tevatron data (magenta) and the region that will be probed by the Tevatron in the near future (red). The blue points are allowed by all the current experimental constraints. The dashed line is the MSSM result for the given SUSY spectrum.

$180-190 \mathrm{GeV}$ ) represent the future Tevatron reach of the $h \rightarrow W^{+} W^{-}$channel. Notice also the presence of a thin stripe of red points, with $m_{h}$ around $120 \mathrm{GeV}$, that extends along a wide range of $m_{A}$ : these models can be probed by the $h \rightarrow b \bar{b}$ channel, that is effective only for relatively low values of $m_{h}$. No points are excluded by the $H \rightarrow b \bar{b}$ decay mode, since $H$ is always much heavier than $120 \mathrm{GeV}$.

The two blue stripes correspond to points where there is no reach from the Tevatron in the $W W$ channel. This can be explained either by a low signal due to the reduced branching fraction into gauge bosons, or simply because the $g g$ parton luminosity is not enough to produce such a heavy Higgs boson. Note however that in the high $m_{h}$ blue region the $Z Z \rightarrow 4 l$ channel becomes kinematically accessible, so that this Higgs could be observed in the gold plated four-lepton mode at the LHC. We will postpone further comments on this region to the next section.

Regarding the LEP constraints, one sees that there are a few currently allowed (blue and red) points below the SM LEP bound of $114.4 \mathrm{GeV}[38,39]$. The nonexclusion is due to the fact that the coupling of $h$ to the gauge bosons is reduced with respect to the SM value. However, all the red points below the LEP-bound can potentially be excluded in the $H \rightarrow W W$ channel.

For the remaining points $\left(m_{A}<160 \mathrm{GeV}\right.$, $114.4 \mathrm{GeV} \lesssim m_{h} \lesssim 170 \mathrm{GeV}$ ), the situation is more complex, and magenta, blue and red points coexist in this region. In particular, there is a region of allowed (blue) points with $m_{h} \sim 130 \mathrm{GeV}-140 \mathrm{GeV}$ and relatively low $m_{A}$. These points have suppressed branching fractions into both $W W$ and $b \bar{b}$, with $A A$ being the dominant decay channel.
In the case of $\tan \beta=20$, the deviations from the MSSM are far less dramatic. Ultimately, this is explained by the fact that several higher-dimension operators are $\tan \beta$ suppressed. However, $m_{h}$ can reach values as high as $160 \mathrm{GeV}$. In this case, since $h$ is SMlike, the LEP bound is very strict, forcing $m_{h}$ to be above $\sim 110 \mathrm{GeV}$. Regarding the Tevatron searches, we see that there are two small and disjoint currently excluded (magenta) regions. The region with $m_{h}$ around $160 \mathrm{GeV}$ corresponds, as in the low $\tan \beta$ case, to exclusion based on the $h \rightarrow W W$ decay mode. The second magenta region has lower values of $m_{h}(114-130 \mathrm{GeV})$ and $m_{A}(100-135 \mathrm{GeV})$. This latter set of models are currently excluded by the inclusive tau search with 2.2 $\mathrm{fb}^{-1}$, using the combination from CDF and D0 [26]. This channel becomes important here, since the $H / A$-and in some cases the $h$-coupling to down-type fermions is $\tan \beta$ enhanced.[? ] Turning to the red points (i.e. those within future Tevatron sensitivity), a closer inspection reveals that all of them can be excluded due to the decay modes of the lightest Higgs. In more detail, the $h \rightarrow b \bar{b}$ channel probes points with $m_{h}$ below $126 \mathrm{GeV}$, while the rest are probed by the $h \rightarrow W W$ search. This can be understood from the fact that, as in the MSSM, in the large $\tan \beta$ limit $H$ tends to be non SM-like. In contrast to the low $\tan \beta$ case, all the Tevatron allowed (blue) points correspond to somewhat heavy values of $m_{A}$ (above $140 \mathrm{GeV}$ ).

It is also interesting to study the relation between the CP-even Higgs masses. In Fig. 2 we show $m_{H}$ as a function of $m_{h}$, for $\tan \beta=2$ (left panel) and $\tan \beta=20$ (right panel). For most of the points these masses are not correlated. For instance, if in the left plot one takes $m_{h}$ in the $120-200 \mathrm{GeV}$ range, then $M_{H}$ can vary between 

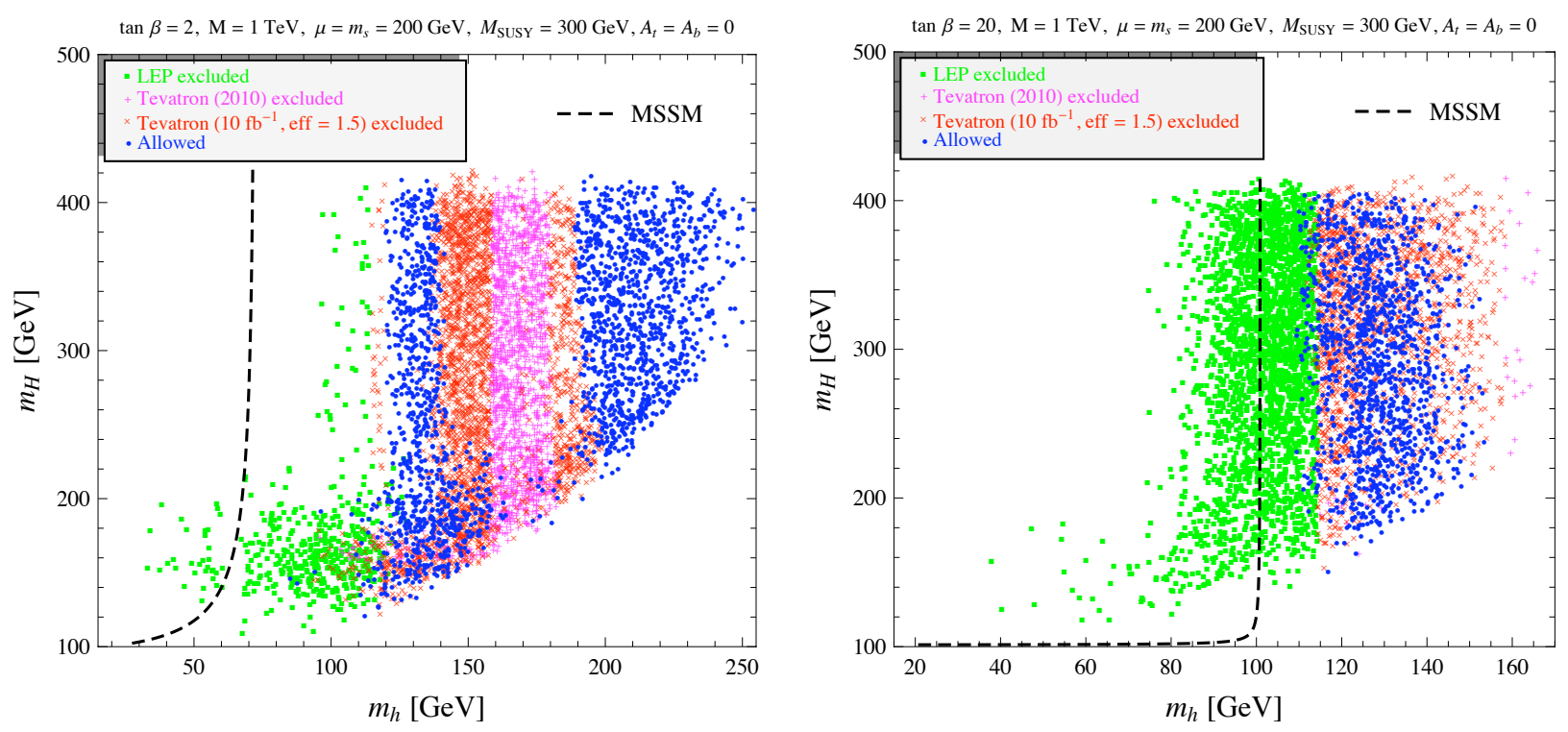

FIG. 2. $m_{H}$ as a function of $m_{h}$, for $\tan \beta=2$ (left panel) and $\tan \beta=20$ (right panel). We show the points excluded by LEP (green), current Tevatron data (magenta) and the region that will be proved by the Tevatron in the near future (red). The blue points are allowed by all the current experimental constraints. The dashed line is the MSSM result for the given SUSY spectrum.

200 and $400 \mathrm{GeV}$. For $\tan \beta=2$, one has not only the (now vertical) stripes corresponding to exclusion due to $h$ that we have found in Fig. 1: there are also horizontal stripes, corresponding to $m_{H}$ ranges where the Tevatron is excluding models by means of the $H \rightarrow W W$ decay channel. This sheds some light into the region already mentioned in the discussion of Fig. 1 with $m_{A}<160 \mathrm{GeV}$ and $114.4 \mathrm{GeV} \lesssim m_{h} \lesssim 170 \mathrm{GeV}$. In this region both $h$ and $H$ can couple to $W W$, typically resulting in some suppression with respect to the SM for one or the other CP-even Higgs boson. This constitutes an interesting example of how the $h$ and $H$ signals can complement each other. The right panel confirms what we have anticipated from our discussion of Fig. 1: in the large $\tan \beta$ regime, $m_{H}$ tends to be heavy, and the decays of $H$ are less restrictive than the ones from $h$, hence there are no horizontal stripes in this plot. As mentioned before, the $h$ search channels give rise to all the red points.

Finally, we show in Fig. 3 the masses of the heavy CPeven and charged Higgs bosons as a function of $m_{A}$. The deviations from the MSSM value are much less dramatic than for $h$. This is particularly true in the large $m_{A}$ limit and for large $\tan \beta$. Nonetheless, in this region the contribution from the new physics effects to the masses is of $\mathcal{O}(10 \mathrm{GeV})$, which cannot be neglected. For low values of $\tan \beta$ (left plots) we see that in the region of blue points with low values of $m_{A}$, both $m_{H^{ \pm}}$and $m_{H}$ are above the MSSM value. Notice that this effect is more important for $m_{H}$ than for $m_{H^{ \pm}}$. As a direct consequence, in the low $\tan \beta$ regime, new exotic channels like $H \rightarrow A A$ and $H^{ \pm} \rightarrow A W^{ \pm}$can be open, with large BRs, as we will see in the next section. This does not happen for $\tan \beta=20$ since, as stated before, there are no allowed (blue and red) points with $m_{A}$ below $140 \mathrm{GeV}$ and the mass splittings do not allow the previous decay modes.

Having analyzed the modifications in the spectra due to the higher-dimension operators, we will devote the next section to study the collider phenomenology of these models.

\section{BMSSM COLLIDER PHENOMENOLOGY}

In this section we study the phenomenology of the BMSSM Higgs sector, including all of the effects and constraints described in Section II. We consider the low and large $\tan \beta$ cases separately.

\section{A. Low $\tan \beta$ searches: general features}

We start with the low $\tan \beta$ regime, fixing $\tan \beta=2$. As we have described in the previous section, the main modification introduced by the higher-dimension operators is to shift the Higgs spectrum with respect to the MSSM one. However, the couplings of the Higgs bosons also get corrected. The combination of these two effects can give rise to sizable modifications in both the Higgs production cross sections and the branching fractions.

We compute the production cross sections in the following way. For the Higgs-strahlung and vector boson fusion processes, we simply scale the corresponding SM cross section by the (square of) the Higgs- $W-W$ coupling in our scenario, normalized to the SM coupling, i.e. by the effective coupling as defined in Eq. (1) (for all practical purposes this ratio coincides with the normalized 

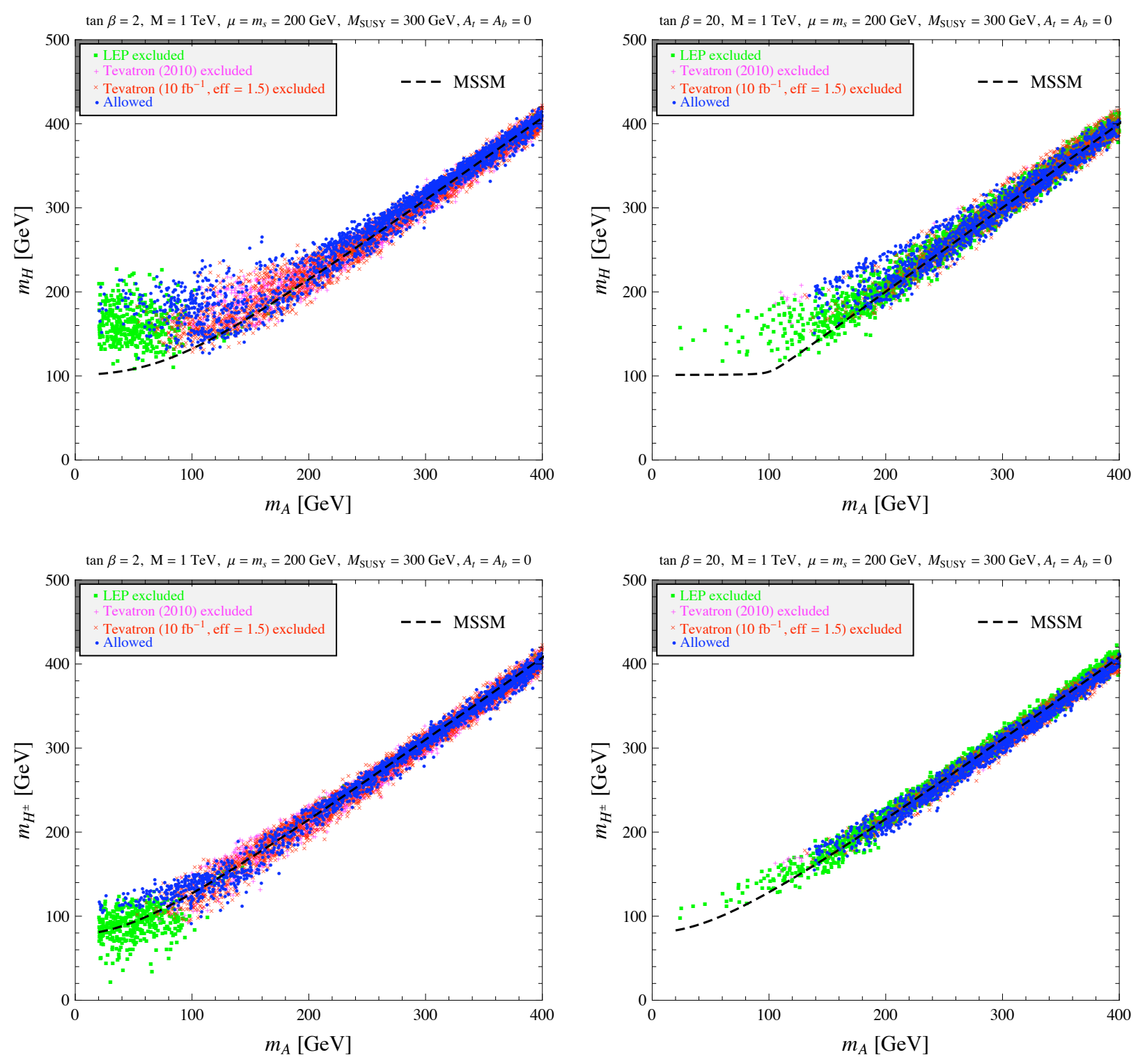

FIG. 3. $H$ (upper row) and $H^{ \pm}$(lower row) masses as a function of $m_{A}$, for $\tan \beta=2$ (left panels) and tan $\beta=20$ (right panels). We show the points excluded by LEP (green), excluded by current Tevatron data (magenta) and the region that will be proved by the Tevatron in the near future (red). The blue points are allowed by all the current experimental constraints. The dashed line is the MSSM result for the given SUSY spectrum.

Higgs- $Z-Z$ coupling [? ]). For the gluon fusion cross section, we shall argue that the NLO K-factor in our scenario is expected to agree with the NLO K-factor in the SM within $20 \%$. This implies that to this accuracy

$$
\frac{\sigma^{\mathrm{NLO}}(g g \rightarrow h)}{\sigma_{\mathrm{SM}}^{\mathrm{NLO}}(g g \rightarrow h)} \approx \frac{\Gamma^{\mathrm{LO}}(h \rightarrow g g)}{\Gamma_{\mathrm{SM}}^{\mathrm{LO}}(h \rightarrow g g)}
$$

since the ratio of cross sections equals the ratio of widths at leading order in $\alpha_{s}[32,40,41]$. The right-hand side of Eq. (2) is computed using our modified version of HDECAY [27], which includes the tree-level expressions for masses and couplings in the presence of the higherdimension operators.
The K-factor in our scenario differs from the SM one in two respects. First, the contribution to the gluon fusion cross section from bottom loops cannot be neglected, specially in the large $\tan \beta$ regime. Second, one has to consider the presence of a relatively light SUSY spectrum. We discuss separately these two effects. To assess the impact of the bottom loop we use the code HIGLU [42], that includes both the LO and NLO results for both the SM and the MSSM [41] (but, at present, does not include SUSY particles in the loop), to compute the K-factors in these two models. We find that at low $\tan \beta$ and for a wide range of Higgs masses, the NLO K-factors for $h, H$ and $A$ coincide within $5 \%$ with the SM NLO K- 
factor for a Higgs of the corresponding mass. At larger $\tan \beta(\sim 30)$ the differences are larger, as expected, but still smaller than about $20 \%$. We expect that the same will hold in our extended SUSY scenarios. The changes in the NLO K-factor due to relatively light sparticles in the loop, again in the MSSM context, were studied in [28], where the effect was found to be less than $3 \%$ for $\tan \beta=1.5$. Therefore, we conclude that at low $\tan \beta$ Eq. (2) holds to an accuracy of better than $10 \%$, and allows us to obtain a sufficiently precise estimate for the NLO gluon fusion cross section in our scenario. Note that this uncertainty is below the one obtained by comparing the NLO and NNLO/NNLL results in the SM calculation [43-48]. It is also important to note that the bulk of the effects of the light SUSY spectrum is taken into account in the LO cross section, and that these effects are fully implemented in HDECAY, which is used to compute the right-hand side of Eq. (2). This also includes radiative effects that correct the bottom Yukawa coupling, which can be important at large $\tan \beta$ [31]. We discuss next a number of general features regarding the BMSSM Higgs signals. In both the SM and the MSSM, the dominant decay channel for a Higgs boson whose mass is greater than $140 \mathrm{GeV}$ is into $\mathrm{W}$ pairs. Therefore, an important observable at a hadron collider is the production cross section times the branching fraction in the WW channel. In Fig. 4 we show this quantity for $h$ and $H$, normalized to the SM result, as a function of the corresponding Higgs mass. In the left panel we clearly see the Tevatron exclusion in the $h \rightarrow W W$ channel: the V-shaped magenta and red regions around $m_{h} \sim 160 \mathrm{GeV}$ correspond to the stripes that were already discussed in Section III (see Figs. 1 and 2). We stress that the blue points with $m_{h}$ above $180 \mathrm{GeV}$ have a slightly enhanced $W W$ signal compared to the SM. In turn, this mass range will be explored at the LHC via the $h \rightarrow Z Z \rightarrow 4 l$ channel (recall that, for all practical purposes, the CP-even Higgs normalized couplings to $W W$ and $Z Z$ are the same, hence the plot can be directly applied to the $Z Z$ channel). Thus, an enhanced signal in this region is an interesting feature: for these points, the Higgs cannot escape detection. For the blue points with $W W$ signal reduced by a factor of 10 or more $\left(m_{h}<160 \mathrm{GeV}\right)$, one may have to rely on other search channels.

Note that this figure exhibits currently allowed (blue and red) points with $m_{h}$ below the LEP bound of $114.4 \mathrm{GeV}$. These correspond to models where the coupling to gauge bosons is below the SM value. We also notice a group of red points whose signal is around the SM value, and with a mass slightly above the LEP bound $\left(114.4 \mathrm{GeV} \leq m_{h} \lesssim 120 \mathrm{GeV}\right)$ : these are within the Tevatron reach in the $h \rightarrow b \bar{b}$ channel, assuming an accumulated luminosity of $10 \mathrm{fb}^{-1}$ per experiment and a $50 \%$ efficiency improvement in this channel.

Turning our attention to the right panel of Fig. 4, we see that in the case of $H$ it is hard to differentiate regions where a single color is predominant, as was possible in the left panel. We can identify a mostly green region with $m_{H}$ above the LEP bound (and above the MSSM curve). These points are excluded by the LEP bound on $m_{h}$ rather than on $m_{H}$, and serve as a reminder that the constraints may come from observables not related to those shown in a given plot. This is not to say that there are no points where the exclusion is through $H$ directly instead of $h$ : for instance, the magenta and red points in the upper left side of the plot correspond to the V-shape exclusion from $H \rightarrow W W$ at the Tevatron. This is the only region where the signal is enhanced with respect to both the SM and the MSSM. These correspond to models where $H$ is SM-like, while $h$ decays mainly into $b \bar{b}$ and $\tau \bar{\tau}$.

Aside from the $W W$ channel, there are other important decay modes for light Higgs bosons, in particular $b \bar{b}, \tau \bar{\tau}$ and $\gamma \gamma$. In the first case, the huge QCD backgrounds render this channel very difficult to measure at a hadron collider. This does not mean, however, that this decay mode is completely useless. For instance, in the Higgstrahlung process, $q \bar{q} \rightarrow Z^{*} / W^{*} \rightarrow Z / W+$ Higgs, the gauge boson can be fully reconstructed from its decay modes, and then Higgs $\rightarrow b \bar{b}$ becomes a feasible option. Another example to search for a SM-like Higgs boson decaying into $b \bar{b}$ is the Higgs associated production together with a top quark pair. This has the problem of being quite challenging at hadron colliders. The di-photon channel, on the other hand, constitutes the most promising decay channel for a relatively light SMlike Higgs at the LHC since, in spite of its tiny BR of $\mathcal{O}\left(10^{-3}\right)$, an excellent energy resolution can be achieved and the background is under good experimental control. The other important search channel at the LHC in the low Higgs mass range is the vector boson fusion with the subsequent decay of the Higgs into a $\tau \bar{\tau}$ pair.

In Fig. 5 we show the branching fraction of $h$ into $b \bar{b}$ (left plot) and $\gamma \gamma$ (right plot), for $\tan \beta=2$. Notice that the $b \bar{b}$ channel can be suppressed with respect to the $\mathrm{SM}$ one, as in the blue points with masses in the $120-150 \mathrm{GeV}$ range and $\operatorname{BR}(h \rightarrow b \bar{b})<10^{-1}$. This is an interesting feature, since it can lead to enhancements in other search channels. One can also see currently allowed (blue and red) points with BRs into $b \bar{b}$ above the SM curve: those have a reduced BR into W's, as we have previously identified in Fig. 4. In the case of $H$ (not shown here), the BR into $b \bar{b}$ is typically higher than the SM value. With respect to the MSSM, we find that there is no definite tendency: $\operatorname{BR}(H \rightarrow b \bar{b})$ can be either increased or suppressed by an order of magnitude. It is worth mentioning that the branching fraction in the $\tau \bar{\tau}$ channel follows closely the $b \bar{b}$ behavior. This is as expected, since the extended Higgs sectors under consideration do not distinguish between the down-type fermions, in the sense that the Yukawa coupling normalized to the $\mathrm{SM}$ value is the same for bottoms and taus, while differences due to the SUSY QCD and top Yukawa interactions, that arise at loop level, are not significant at small $\tan \beta$.

Turning our attention to the right panel of Fig. 5, 

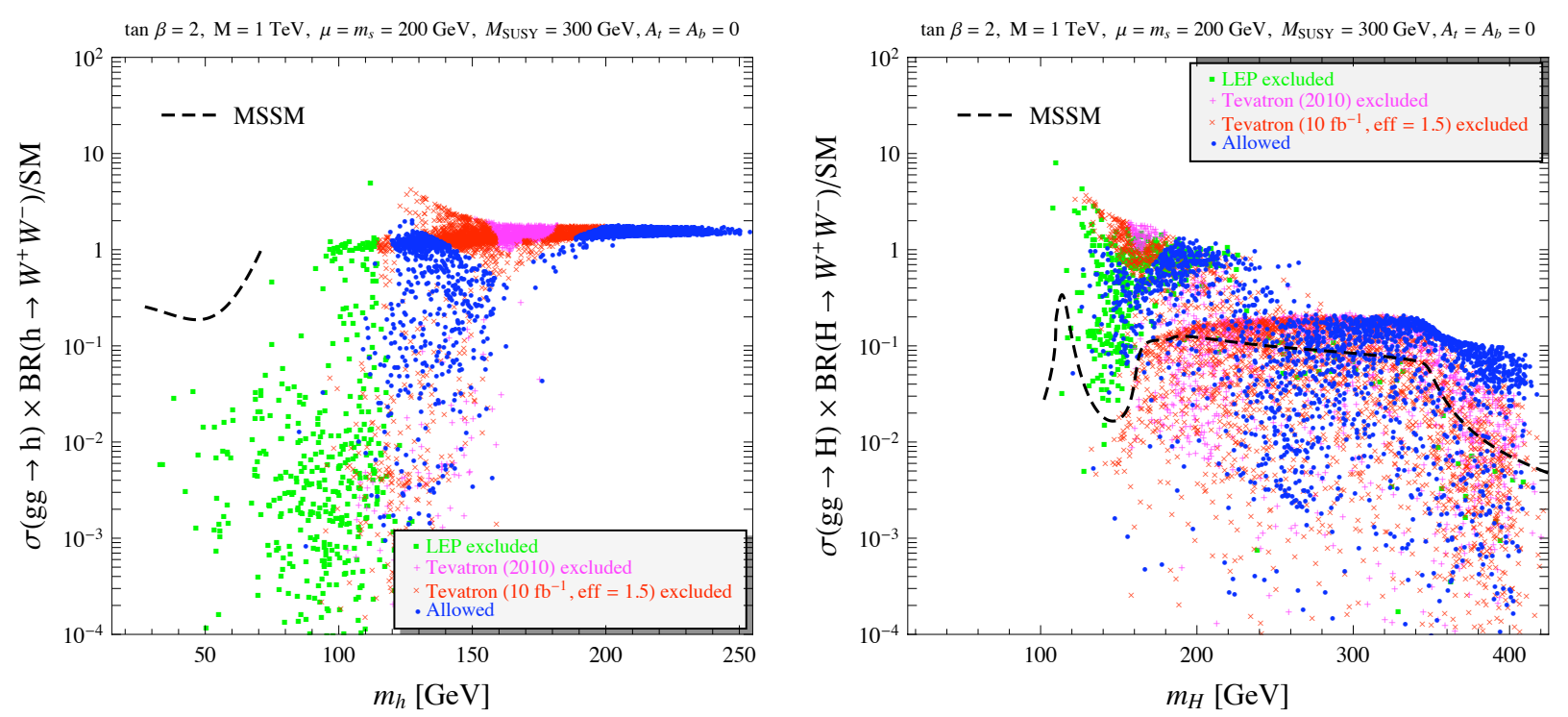

FIG. 4. Production cross section by gluon fusion times branching ratio into $W$ boson pairs, for $h$ (left panel) and $H$ (right panel), normalized to the SM result, as a function of the corresponding Higgs mass, for $\tan \beta=2$. We show the points excluded by LEP (green), excluded by current Tevatron data (magenta) and the region that will be probed by the Tevatron in the near future (red). The blue points are allowed by all the current experimental constraints. The dashed line corresponds to the MSSM prediction for the given SUSY spectrum.
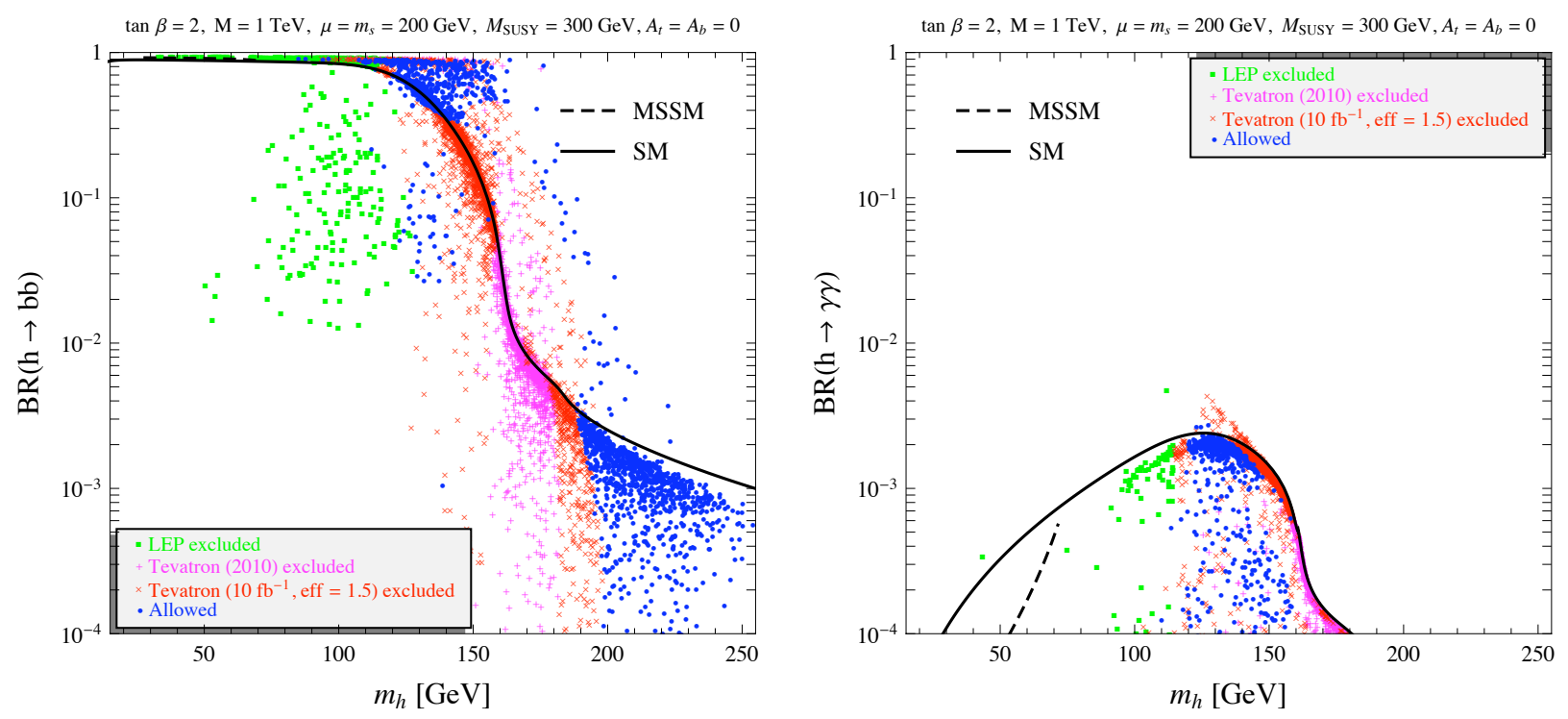

FIG. 5. Branching fractions for $h \rightarrow b \bar{b}$ (left panel) and $h \rightarrow \gamma \gamma$ (right panel) for $\tan \beta=2$. We show the points excluded by LEP (green), excluded by current Tevatron data (magenta) and the region that will be probed by the Tevatron in the near future (red). The blue points are allowed by all the current experimental constraints. The solid (dashed) line corresponds to the SM (MSSM) result.

we see that most models present a suppressed branching fraction in the di-photon channel. However, it is worth noticing the group of points above the SM curve, where an enhancement of up to a factor of 2 can be achieved.

Decays of the CP-even Higgs bosons into pairs of $A$ bosons can become the dominant decay mode. Such a scenario has been previously considered in the literature (see, for instance, [49] for a model-independent analysis, and [50] for NMSSM studies). In Fig. 6 we show the branching fraction of $h$ and $H$ into $A A$, for $\tan \beta=2$. The left panel shows that the branching fraction in this channel can reach $\mathcal{O}(1)$ values, thus becoming the most relevant decay mode of $h$. The Tevatron allowed (blue) points in this figure present a reduced branching fraction in both the $b \bar{b}$ and the $W W$ channel, and were already mentioned in the context of Fig. 5. In the case of $H$ (right panel), the branching fractions vary considerably, but the $A A$ channel may still become the primary decay 

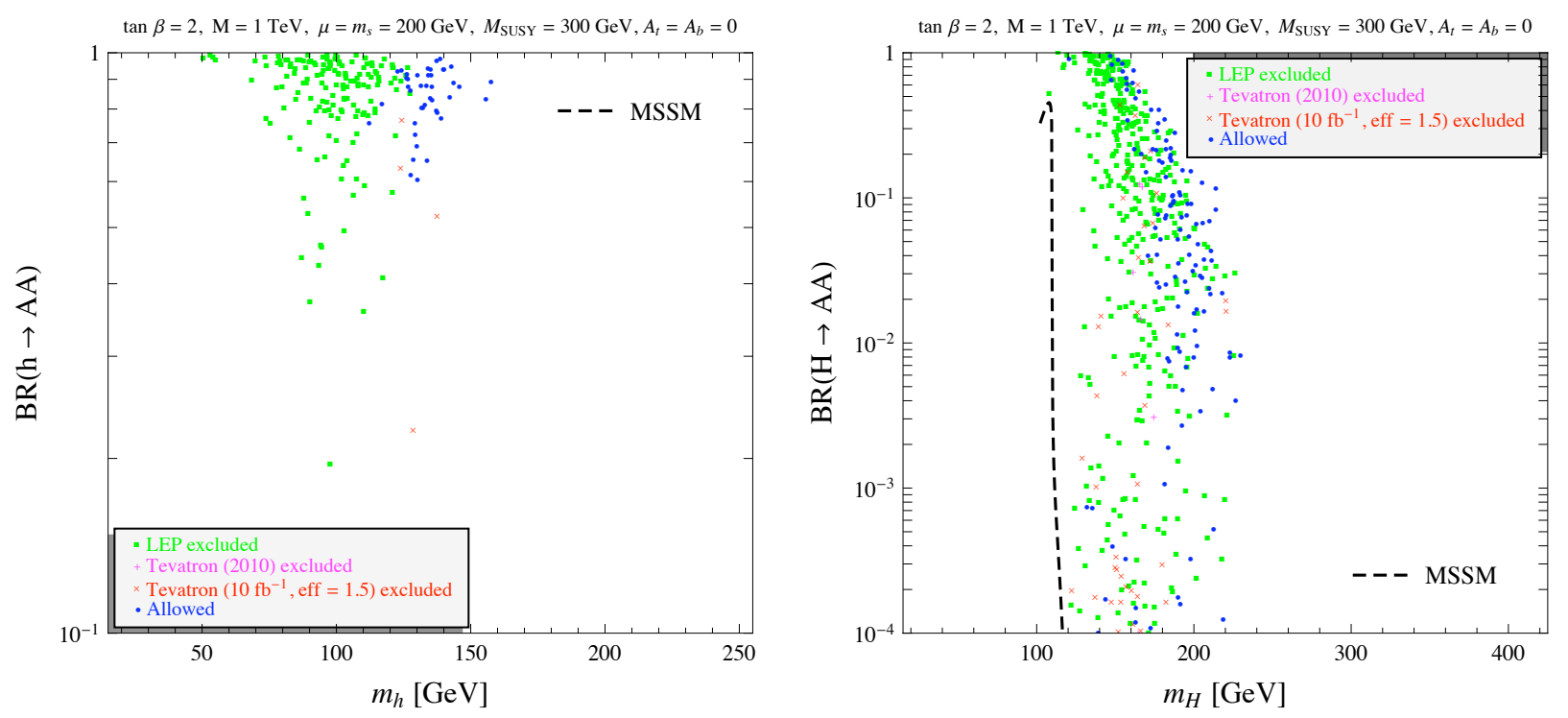

FIG. 6. Branching fractions for $h \rightarrow A A$ (left plot) and $H \rightarrow A A$ (right plot), for $\tan \beta=2$. We show the points excluded by LEP (green), excluded by current Tevatron data (magenta) and the region that will be proved by the Tevatron in the near future (red). The blue points are allowed by all the current experimental constraints. The dashed line corresponds to the MSSM result for the given SUSY spectrum.

mode in some models.

The main modification to the decay phenomenology of $A$ and $H^{ \pm}$with respect to the MSSM is due to the shift in the overall Higgs spectrum. The channels that change the most are those that involve a Higgs decaying into either a pair of Higgs bosons, or a Higgs boson plus a gauge boson. As an example of the latter, we take the decay of $H^{ \pm}$into a W boson and a neutral Higgs. In the MSSM, since $h$ tends to be rather light, one has that $H^{ \pm} \rightarrow h W^{ \pm}$ can be an important decay channel. On the other hand, since $m_{A}$ and $m_{H^{ \pm}}$tend to be rather degenerate, one finds that $H^{ \pm} \rightarrow A W^{ \pm}$is generally highly suppressed. On the contrary, in the context of the BMSSM, one can find points where the mass hierarchy suffers an inversion, i.e. $m_{A}$ can be well below $m_{h}$ and split from $m_{H^{ \pm}}$. In this case, one finds that $A$ and $h$ interchange their roles with respect to the above described situation in the MSSM, as can be seen in Fig. 7. The left panel shows points where the $A W^{ \pm}$channel has a $\mathrm{BR}$ greater than 0.1 , while the right panel shows that the $h W^{ \pm}$decay mode is highly suppressed. In this case, the process $H^{ \pm} \rightarrow A W^{ \pm} \rightarrow$ $b \bar{b} W^{ \pm}$can give rise to an interesting signal at the LHC, possibly allowing the discovery of two nonstandard Higgs bosons. Note that we have already encountered another example of an inversion between $A$ and $h$ in the context of Fig. 6: the potentially open MSSM channels $A \rightarrow h h$ and $H \rightarrow h h$ are replaced by $h \rightarrow A A$ and $H \rightarrow A A$ in the BMSSM context.

Finally, turning our attention to $A$, we have found that the $A \rightarrow h Z$ decay channel is significantly reduced with respect to the MSSM value $\sim 0.3$ for values of $m_{A}$ below $250 \mathrm{GeV}$, due to the shift in $m_{h}$ that disfavors this decay mode. [? ] This reduction brings an enhancement in both the $b \bar{b}$ and $\tau \bar{\tau}$ channels. As in the MSSM, the former is the dominant decay channel below the $t \bar{t}$ threshold, and the latter stays almost constant at about $10 \%$.

\section{B. Low $\tan \beta$ searches: benchmark points}

Up to this point we have analyzed each observable almost independently of the others. We would like to understand, however, how the different features that we have singled out are correlated with each other. We shall consider benchmark scenarios currently allowed by LEP and Tevatron data and explore two possibilities: a) models that can be probed at $95 \%$ C.L at the Tevatron in the near future, from now on referred to as Tevatron covered (red) points, and b) models that are beyond the expected Tevatron reach and will be explored at the LHC, from now on referred to as Tevatron uncovered (blue) points. We will also indicate the importance of the $1 / M^{2}$ effects, as measured by their impact on $m_{h}$.

\section{Scenarios within the Tevatron reach}

The Tevatron covered models can be divided into three subsets, according to which channel can exclude the point: $h \rightarrow b \bar{b}, h \rightarrow W W$ and $H \rightarrow W W$. It is interesting to ask whether a given model can be probed by more than one channel at the Tevatron. We find, however, that the previous subsets are disjoint. The disjointness between the subsets probed by $h \rightarrow b \bar{b}$ and 

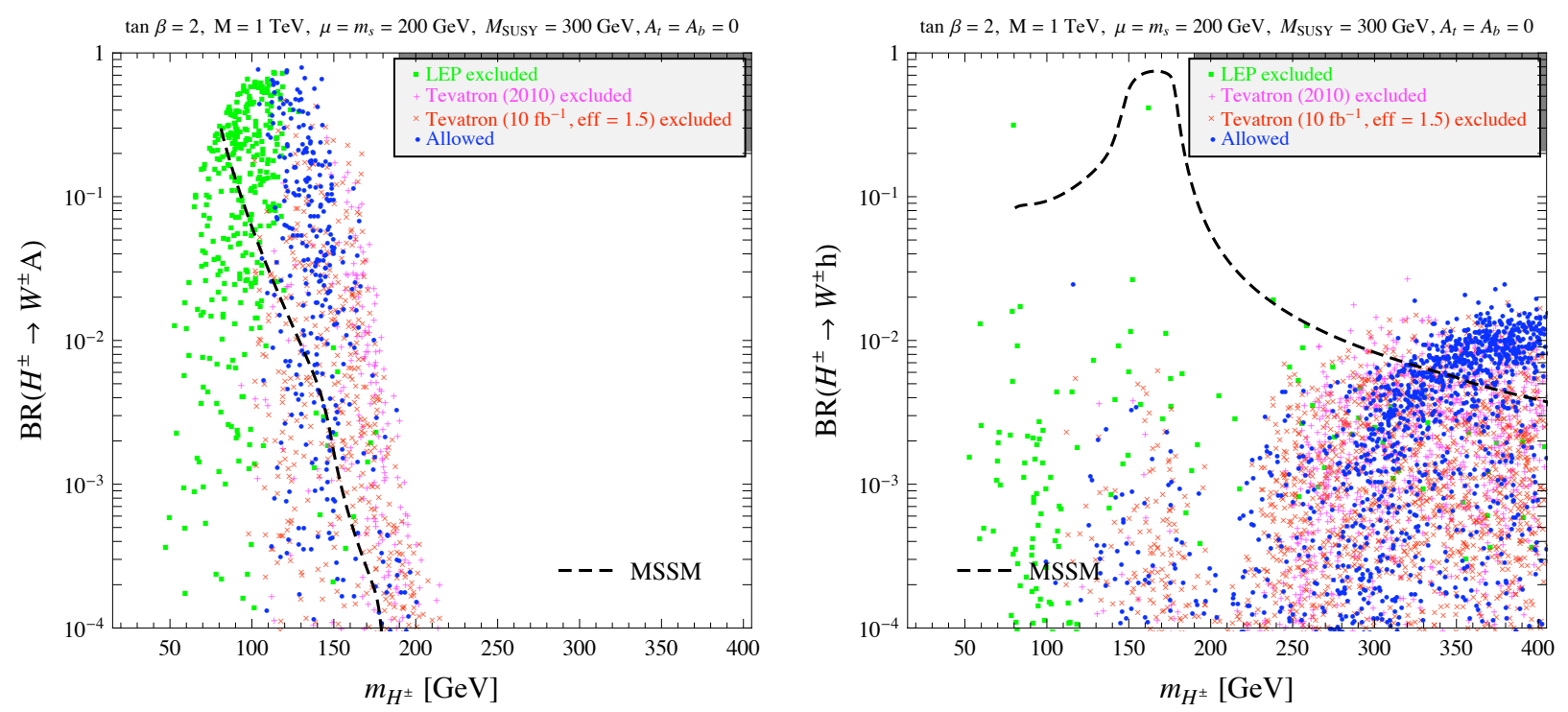

FIG. 7. $H^{ \pm} \rightarrow A W^{ \pm}$(left panel) and $H^{ \pm} \rightarrow h W^{ \pm}$(right panel), for $\tan \beta=2$. The dashed line corresponds to the MSSM result for the given $S U S Y$ spectrum.

$h \rightarrow W W$ can be understood in terms of the relevant mass ranges, since the $b \bar{b}$ search is most sensitive to the $m_{h} \lesssim 120 \mathrm{GeV}$ range, while the di-boson channel probes the region $165 \pm 20 \mathrm{GeV}$. In principle, they do not have to be mutually exclusive, but one would need an enhancement of 3.4 over the SM in the $h \rightarrow W W$ signal in order to probe a $120 \mathrm{GeV}$ Higgs in this channel,[? ] which is not achievable within these models: the increase in the $g g \rightarrow h \rightarrow W W$ cross section is always below a factor of 2 . In the case of $h$ and $H$ decaying into $\mathrm{W}$ bosons, even if both of them are in the favorable mass region $(\sim 150-170 \mathrm{GeV})$, the MSSM sum rule $g_{h W W}^{2}+g_{H W W}^{2}=1$ is valid within $5 \%$ accuracy, and it is not possible for $h$ and $H$ to have large enough couplings to W's for both signals to simultaneously be within the Tevatron reach. The subsets probed by the $h \rightarrow b \bar{b}$ and $H \rightarrow W W$ searches are disjoint because both processes require a sizable coupling of the Higgs to $W$ 's (for production and decay, respectively), but this does not happen when $m_{h}$ and $m_{H}$ are sufficiently different, as would be required for simultaneous searches in these two channels.

\section{Point A: MSSM-like scenarios}

We start our analysis with the points that can be probed via the $h \rightarrow b \bar{b}$ decay mode. We find that these do not differ greatly from the decoupling limit of the MSSM with rather heavy sparticles $(\sim$ a few $\mathrm{TeV})$. In this case, the observation of a light SUSY spectrum (in the few hundred $\mathrm{GeV}$ range) would be the smoking gun of BMSSM physics, since such a light SUSY spectrum would be in conflict with the LEP limits on the MSSM lightest CP-even Higgs boson. We illustrate the main features of this subset by showing point A [? ] in Table I, where we include the mass spectrum and the branching
POINT A

\begin{tabular}{|c|c|c|c|}
\hline$m_{A}(\mathrm{GeV})$ & $m_{h}(\mathrm{GeV})$ & $m_{H}(\mathrm{GeV})$ & $m_{H^{ \pm}}(\mathrm{GeV})$ \\
\hline 239 & 118 & 246 & 245 \\
\hline$g_{h W W}^{2}$ & $g_{H W W}^{2}$ & $g_{h g g}^{2}$ & $g_{H g g}^{2}$ \\
\hline 0.992 & 0.008 & 1.06 & 0.55 \\
\hline channel & BMSSM (SM) & channel & BMSSM (SM) \\
\hline$h \rightarrow b \bar{b}$ & $0.78(0.73)$ & $h \rightarrow W W$ & $0.08(0.11)$ \\
$h \rightarrow \tau \bar{\tau}$ & $0.08(0.08)$ & $h \rightarrow \gamma \gamma / 10^{-3}$ & $1.42(2.30)$ \\
$H \rightarrow b \bar{b}$ & 0.15 & $H \rightarrow W W$ & 0.22 \\
$H \rightarrow Z Z$ & 0.11 & $H \rightarrow h h$ & 0.50 \\
$A \rightarrow b \bar{b}$ & 0.89 & $H^{+} \rightarrow t \bar{b}$ & 0.99 \\
$A \rightarrow \tau \bar{\tau}$ & 0.08 & $A \rightarrow Z h$ & 0.24 \\
\hline
\end{tabular}

TABLE I. Masses and branching fractions in the BMSSM (and in the $S M$ for $h$ ) for point $A$. We only show the main decay modes. The effective couplings $g_{\phi X}^{2}$ were defined in Eq. (1).

fractions of the most important decay channels for each Higgs boson. For reference, in the case of $h$ we also indicate between parentheses the SM values. We also note that for this point, the $1 / M^{2}$ operators contribute about $25 \mathrm{GeV}$ to $m_{h}$.

Generically, the branching fractions of $h$ do not deviate much from the SM ones. One finds a small increase in the $h \rightarrow b \bar{b}$ channel, while $\operatorname{BR}(h \rightarrow \gamma \gamma)$ is slightly suppressed with respect to the SM (by at most a factor of $3)$. Since $g_{h W W}^{2}$ and $g_{h g g}^{2}$ are close to one, the production cross sections by Higgs-strahlung and gluon fusion are, for all practical purposes, the same as in the SM. Thus, the change in the signal is given by the ratio of the branching fractions in our scenario to those in the SM. For point A, the production rate in $h \rightarrow b \bar{b}$ is $6 \%$ 
POINT B

\begin{tabular}{|c|c|c|c|}
\hline$m_{A}(\mathrm{GeV})$ & $m_{h}(\mathrm{GeV})$ & $m_{H}(\mathrm{GeV})$ & $m_{H}(\mathrm{GeV})$ \\
\hline 101 & 129 & 141 & 135 \\
\hline$g_{h W W}^{2}$ & $g_{H W W}^{2}$ & $g_{h g g}^{2}$ & $g_{H g g}^{2}$ \\
\hline 0.8 & 0.2 & 1.72 & 0.06 \\
\hline channel & BMSSM (SM) & channel & BMSSM (SM) \\
\hline$h \rightarrow b \bar{b}$ & $0.01(0.56)$ & $h \rightarrow \tau \bar{\tau}$ & $0.001(0.06)$ \\
$h \rightarrow W W$ & $0.63(0.28)$ & $h \rightarrow Z Z$ & $0.08(0.04)$ \\
$h \rightarrow$ jets & $0.26(0.06)$ & $h \rightarrow \gamma \gamma / 10^{-3}$ & $3.97(2.38)$ \\
$H \rightarrow b \bar{b} / \tau \bar{\tau}$ & $0.84 / 0.09$ & $H \rightarrow W W$ & 0.05 \\
$A \rightarrow b \bar{b} / \tau \bar{\tau}$ & $0.89 / 0.09$ & $H^{+} \rightarrow \tau \nu_{\tau}$ & 0.87 \\
\hline
\end{tabular}

TABLE II. Masses and branching fractions in the BMSSM (and in the $S M$ for $h$ ) for point $B$.

above the SM result. In this case, the Tevatron could claim a hint on a SM Higgs boson, while at the LHC the direct detection of $h$ would proceed in the di-photon channel, since the $g g \rightarrow h \rightarrow \gamma \gamma$ cross section is 0.65 of the SM value. Some of the remaining Higgs bosons may also be observed. For $H$ and $A$, the $H \rightarrow h h \rightarrow \gamma \gamma b \bar{b}$ and $A \rightarrow Z h \rightarrow l l b \bar{b}$ searches provide the best prospects for discovery [52]. For a charged Higgs with a mass above $m_{t}$, the ATLAS update of 2009 [53] found that the $t b$ channel is rather challenging and that the low $\tan \beta$ region cannot be covered.

\section{Point B: Light Higgs spectra}

We turn now our attention to the models that can be excluded at the Tevatron by the $h \rightarrow W W$ channel. Those can be further split into two categories, according to whether $m_{h}$ is high $(\gtrsim 170 \mathrm{GeV})$ or low $(\lesssim 160 \mathrm{GeV})$, corresponding to the two red stripes defined in the context of Fig. 1. As a general feature of the lower red stripe, the branching fraction of $h$ into $b \bar{b}$ can be sizably reduced with respect to the $\mathrm{SM}$, as we pointed out in the left panel of Fig. 5. This implies that the remaining channels are enhanced, which is interesting for the $h \rightarrow W W$ and $h \rightarrow \gamma \gamma$ decay modes. We present as an example point B [? ] in Table II. Here, one sees that the Higgs spectrum is relatively light. $H$ is the heaviest Higgs, while $h$ is lighter than $H^{ \pm}$, but heavier than $A$. It turns out that for this point, the $1 / M^{2}$ effects result in a slight net reduction of $m_{h}$ by a couple of $\mathrm{GeV}$.

Since $h$ is SM-like, we give in parentheses the corresponding branching fractions in the SM. Here we clearly observe that $h$ presents an increase in the gluon fusion cross section, and in the branching fractions into photons and $\mathrm{W}$ bosons, accompanied by a sizable reduction in the down-type fermion decay modes. Note that the $g g \rightarrow h \rightarrow \gamma \gamma$ and $g g \rightarrow h \rightarrow W W$ signals are larger than in the SM by factors of 2.86 and 3.82 respectively, which would facilitate the search of $h$ at the LHC as well. $H$ decays mainly into bottoms and taus, and its production cross section by gluon fusion is strongly reduced with respect to the SM case. The most promising discovery channel at the LHC would be $q q H \rightarrow q q \tau \bar{\tau}$, where the
POINT C

\begin{tabular}{|c|c|c|c|}
\hline$m_{A}(\mathrm{GeV})$ & $m_{h}(\mathrm{GeV})$ & $m_{H}(\mathrm{GeV})$ & $m_{H^{ \pm}}(\mathrm{GeV})$ \\
\hline 135 & 174 & 186 & 164 \\
\hline$g_{h W W}^{2}$ & $g_{H W W}^{2}$ & $g_{h g g}^{2}$ & $g_{H g g}^{2}$ \\
\hline 0.11 & 0.89 & 1.05 & 0.65 \\
\hline channel & BMSSM (SM) & channel & BMSSM (SM) \\
\hline$h \rightarrow b \bar{b}$ & $0.12(0.01)$ & $h \rightarrow W W$ & $0.84(0.96)$ \\
$H \rightarrow W W$ & $0.81(0.82)$ & $H \rightarrow Z Z$ & $0.17(0.17)$ \\
$A \rightarrow b \bar{b}$ & 0.90 & $A \rightarrow \tau \bar{\tau}$ & 0.10 \\
$H^{+} \rightarrow \tau \nu_{\tau}$ & 0.59 & $H^{+} \rightarrow t \bar{b}$ & 0.38 \\
\hline
\end{tabular}

TABLE III. Masses and branching fractions in the BMSSM (and in the $S M$ for $h$ and $H$ ) for point $C$.

signal is reduced with respect to the SM by a factor of two. The CP-odd $A$ decays as in the MSSM, while for the charged Higgs the $\tau \nu_{\tau}$ channel is the dominant one.

Point C: The heavy CP-even $H$ as the SM-like Higgs

In the high $m_{h}$ region that can be probed at the Tevatron in the $h \rightarrow W W$ channel one finds an unusual SUSY spectrum. Typically, one runs into the previously mentioned inversions between $h$ and $A$. Moreover, $h$ can also be heavier than the charged Higgs, which is a feature that is not present in the region where $m_{h}$ is below $150 \mathrm{GeV}$. We illustrate this with point $\mathrm{C}$ [? ] in Table III. For this point, the $1 / M^{2}$ operators contribute about $30 \mathrm{GeV}$ to $m_{h}$.

Here we see that the two CP-even Higgs bosons have masses well above the maximum value for $m_{h}$ that can be obtained in the $m_{h}$ max scenario within the MSSM context. In this case, it makes sense to compare both $h$ and $H$ with the SM Higgs. For this particular point, $h$ has not been yet excluded by the Tevatron search since it is not SM-like and its branching fraction into $W W$ is somewhat suppressed. $H$ can be discovered by the LHC in the $Z Z \rightarrow 4 l$ mode, since the signal normalized to the $\mathrm{SM}$ value, is 0.65 . We note that here both $m_{h}$ and $m_{H}$ are near the region where the $W W$ channel opens up leading to a suppression in the sensitivity of the $Z Z$ search mode at the LHC. We recall that such a heavy SM-like $H$ is not a feature of the MSSM, being a unique characteristic of the BMSSM Higgs sector. The CP-odd $A$ decays almost entirely to bottom and tau pairs, while the charged Higgs has sizable decays into both the $\tau \nu_{\tau}$ and $t \bar{b}$ channels.

The last subset of the Tevatron covered points corresponds to those than can be probed by the $H \rightarrow W W$ search, for which the $g g \rightarrow H \rightarrow W W$ signal goes between $0.4-4$ times the SM value. In such scenarios, $h$ and $A$ decay mostly into bottoms and taus. In some cases the $h \rightarrow \gamma \gamma$ or $q q H \rightarrow q q \tau \tau$ signal might be observable at the LHC. The charged Higgs is relatively light (always below $200 \mathrm{GeV}$ ) and will decay almost $100 \%$ of the time into $\tau \nu_{\tau}$ for masses below $160 \mathrm{GeV}$, and in $t \bar{b}$ for the remaining points. The $H^{ \pm} \rightarrow h W^{ \pm}$decay mode is closed for kinematical reasons, as we already know from 
POINT D

\begin{tabular}{|c|c|c|c|}
\hline$m_{A}(\mathrm{GeV})$ & $m_{h}(\mathrm{GeV})$ & $m_{H}(\mathrm{GeV})$ & $m_{H^{ \pm}}(\mathrm{GeV})$ \\
\hline 184 & 204 & 234 & 203 \\
\hline$g_{h W W}^{2}$ & $g_{H W W}^{2}$ & $g_{h g g}^{2}$ & $g_{H g g}^{2}$ \\
\hline 0.3 & 0.7 & 1.39 & 0.36 \\
\hline channel & BMSSM (SM) & channel & BMSSM (SM) \\
\hline$h \rightarrow W W$ & $0.73(0.72)$ & $h \rightarrow Z Z$ & $0.25(0.27)$ \\
$H \rightarrow W W$ & $0.70(0.71)$ & $H \rightarrow Z Z$ & $0.29(0.29)$ \\
$A \rightarrow b \bar{b}$ & 0.87 & $H^{+} \rightarrow t \bar{b}$ & 0.99 \\
\hline \multicolumn{3}{|c}{} \\
\hline
\end{tabular}

TABLE IV. Masses and branching fractions in the BMSSM (and in the $S M$ for $h$ and $H$ ), for point $D$.

Fig. 7. In addition, when $m_{A}$ is light, the $h \rightarrow A A$ and $H^{ \pm} \rightarrow A W^{ \pm}$channels might become important. Since this also happens with the Tevatron uncovered points, we will defer further comments and the study of a suitable benchmark point for the next subsection.

\section{LHC searches}

Regarding the Tevatron uncovered points, we can also split them into two disjoint subsets, corresponding to each of the blue stripes in Figs. 1 or 2: we will refer to them as low mass $\left(m_{h} \lesssim 140 \mathrm{GeV}\right)$ and high mass (above $190 \mathrm{GeV}$ ) regions. In the high $m_{h}$ case, one can make a further distinction according to whether $m_{A}$ is below or above $160 \mathrm{GeV}$. Again, we illustrate the possibilities with a few benchmark points.

\section{Point D: Two peaks in the $Z Z \rightarrow 4 l$ signal}

General features of the high $m_{h}$, high $m_{A}$ case are: increased $g g \rightarrow h$ cross section with respect to the SM, and negligible (below 2\%) changes in the $h \rightarrow W W / Z Z$ decay modes. Regarding $H$, one has that the signal in the $g g \rightarrow H \rightarrow W W / Z Z$ channel is always suppressed with respect to the SM. As an example, we show point D [?] in Table IV. Here, the $1 / M^{2}$ operators contribute about $40 \mathrm{GeV}$ to $m_{h}$. Given the features of this point, it again makes sense to compare both CP-even Higgs bosons with the SM.

The rise in the mass of $h$ automatically closes the $H \rightarrow h h, A \rightarrow h Z$ and $H^{ \pm} \rightarrow h W^{ \pm}$decay modes, which could be important in the MSSM case. This picture can suffer some alterations if $h$ stays around $200 \mathrm{GeV}$, while the rest of the Higgs bosons attain values around $400 \mathrm{GeV}$, since this will open not only the previously mentioned channels, but possibly also decays into sparticles. In such a case, one would run into a sort of $M S S M$ decoupling limit, but with a mass for the lightest Higgs which is unattainable within the MSSM. Concentrating on point $\mathrm{D}$, we emphasize that both $h$ and $H$ couple in a sizable way to the electroweak gauge bosons, and thus the measurement of both couplings will permit a detailed study of the EWSB mechanism, as it arises from a 2HDM. One possibility to discover these CP-even Higgs
POINT E

\begin{tabular}{|c|c|c|c|}
\hline$m_{A}(\mathrm{GeV})$ & $m_{h}(\mathrm{GeV})$ & $m_{H}(\mathrm{GeV})$ & $m_{H^{ \pm}}(\mathrm{GeV})$ \\
\hline 134 & 181 & 205 & 165 \\
\hline$g_{h W W}^{2}$ & $g_{H W W}^{2}$ & $g_{h g g}^{2}$ & $g_{H g g}^{2}$ \\
\hline 0.03 & 0.95 & 0.79 & 0.99 \\
\hline channel & BMSSM (SM) & channel & BMSSM (SM) \\
\hline$h \rightarrow b \bar{b}$ & $0.23(0.005)$ & $h \rightarrow \tau \bar{\tau}$ & $0.03(0.0005)$ \\
$h \rightarrow W W$ & $0.68(0.92)$ & $h \rightarrow Z Z$ & $0.04(0.07)$ \\
$H \rightarrow W W$ & $0.72(0.73)$ & $H \rightarrow Z Z$ & $0.27(0.27)$ \\
$A \rightarrow b \bar{b}$ & 0.89 & $A \rightarrow \tau \bar{\tau}$ & 0.10 \\
$H^{+} \rightarrow t \bar{b}$ & 0.57 & $H^{+} \rightarrow \tau \nu_{\tau}$ & 0.40 \\
\hline \multicolumn{4}{|r}{} \\
\hline
\end{tabular}

TABLE V. Masses and branching fractions (and in the SM for $h$ and $H$ ) for point $E$.

bosons would be to search for two isolated peaks in the $Z Z \rightarrow 4 l$ golden mode. Notice that the branching fractions of both $h$ and $H$ are very close to their SM counterparts, while there is a difference in the gluon fusion production cross section. Since the $g g \rightarrow H \rightarrow Z Z$ signal can be sizably suppressed with respect to the SM, the direct detection of $H$ in this channel might not be feasible. Nevertheless, a large number of models similar to point $\mathrm{D}$ would in fact present two clear peaks in the di-lepton invariant mass distribution. We also note that these points have a $g g \rightarrow h \rightarrow W W / Z Z$ signal that can be $20-70 \%$ larger than the SM value.

Point E: Non-SM-like Higgs with a clear di-boson signal

One interesting example of a point where $m_{h}$ is still high, but $m_{A}$ is below $160 \mathrm{GeV}$ is given in point $\mathrm{E}$ [? ] , shown in Table $\mathrm{V}$. We see that this point has an unusual Higgs hierarchy, since here $h$ is heavier than both $A$ and $H^{ \pm}$(in this respect similar to point $\mathrm{C}$ ). The signal for $h \rightarrow W W$, normalized to the $\mathrm{SM}$, is 0.54 . The CP-even $H$ will be discovered first and will appear to be the SM Higgs, since the signal is very close to the $\mathrm{SM}$ one. Soon after, $h$ will be found in both the $Z Z$ and $W W$ channels, thus providing a clear evidence of new physics. Notice that here the coupling of $h$ to gauge bosons is extremely small, but due to kinematics it still decays preferentially into gauge bosons. We stress again that this is a unique characteristic of the BMSSM Higgs sector in the low $\tan \beta$ regime, since in the MSSM a similar behavior can only occur for $H$. For this point, the $1 / M^{2}$ operators contribute about $30 \mathrm{GeV}$ to $m_{h}$.

\section{Point F: Multi-Higgs decay chains}

For the points outside the Tevatron reach with $m_{h}$ below $140 \mathrm{GeV}$, the most remarkable feature is the possibility of having the channels $h \rightarrow A A$ and $H \rightarrow A A$ kinematically open. For the points where these channels are closed, the situation is not as interesting, so we will focus on the first scenario. As an example, we show point $F[?]$ in Table VI, where these channels are the dominant decay modes of both $h$ and $H$. For this point, the $1 / M^{2}$ operators contribute about $10 \mathrm{GeV}$ to $m_{h}$. Focusing on the $A A$ channel, the possible final 


\section{POINT F}

\begin{tabular}{|c|c|c|c|}
\hline$m_{A}(\mathrm{GeV})$ & $m_{h}(\mathrm{GeV})$ & $m_{H}(\mathrm{GeV})$ & $m_{H^{ \pm}}(\mathrm{GeV})$ \\
\hline 64 & 135 & 155 & 125 \\
\hline$g_{h W W}^{2}$ & $g_{H W W}^{2}$ & $g_{h g g}^{2}$ & $g_{H g g}^{2}$ \\
\hline 0.002 & 0.991 & 0.65 & 1.17 \\
\hline channel & BMSSM & channel & BMSSM \\
\hline$h \rightarrow b \bar{b}$ & 0.15 & $h \rightarrow A A$ & 0.84 \\
$H \rightarrow W W$ & 0.12 & $H \rightarrow A A$ & 0.84 \\
$H \rightarrow b \bar{b}$ & 0.02 & $A \rightarrow b \bar{b}$ & 0.92 \\
$H^{+} \rightarrow \tau \nu_{\tau}$ & 0.56 & $H^{ \pm} \rightarrow W^{ \pm}+A$ & 0.40 \\
\hline \multicolumn{3}{|c}{} \\
\hline
\end{tabular}

TABLE VI. Masses and branching fractions in the BMSSM for point $F$.

states for $h$ and $H$ are $b \bar{b} b \bar{b}, b \bar{b} \tau \bar{\tau}$ and $\tau \bar{\tau} \tau \bar{\tau}$ [49-51]. The first one is very challenging due to the enormous QCD background, while the third one suffers from a reduced signal $[B R(A \rightarrow \tau \bar{\tau}) \sim 10 \%]$. This leaves the $b \bar{b} \tau \bar{\tau}$ channel as the most promising one. For the case of $H$, one may also look at the $g g \rightarrow H \rightarrow W W$ channel, whose signal is $1 / 5$ of the SM value, and could be discovered with about $100 \mathrm{fb}^{-1}[52]$. For the charged Higgs, the dominant decay mode is $\tau \nu_{\tau}$. Notice also that $H^{ \pm} \rightarrow A W^{ \pm}$can have a sizable branching fraction, offering the possibility to discover both $A$ and $H^{ \pm}$simultaneously in this decay mode.

\section{Large $\tan \beta$ searches: general features}

In this subsection we present our analysis for the large $\tan \beta$ regime, fixing $\tan \beta=20$. As shown in Section III, the changes in the spectrum with respect to the MSSM are less important than in the low $\tan \beta$ case.

We use Eq. (2) to estimate the gluon fusion production cross section at NLO in $\alpha_{s}$. Although the impact of the bottom loop in the $K$-factor is more important for larger $\tan \beta$, the NLO K-factor in our model is still expected to be within $20 \%$ of the NLO SM K-factor, as discussed at the beginning of Section IV A. Furthermore, as shown in [28], the effects on the $\mathrm{K}$ factor due to a light sparticle spectrum like the one we are considering are negligible at large $\tan \beta$. Hence, we conclude that simply computing the right-hand side of Eq. (2) allows us to obtain the NLO gluon fusion production cross section within $20 \%$ accuracy even at large $\tan \beta$. In this regime, production in association with a $b \bar{b}$ pair can become important, and can be obtained in our model from existing results by a simple rescaling with the effective coupling $g_{\phi b b}^{2}$, where $\phi=h, H, A$ [see Eq. (1)].

We show in Fig. 8 the effective coupling of $h$ to downtype fermions, $g_{h b \bar{b}}^{2}$, for both large (left panel) and small (right panel) $\tan \beta$. At large $\tan \beta$, one sees that the currently allowed models (blue and red points) have a gluon fusion production cross section which ranges from $0.7-5$ times the SM value. The most striking feature is that the coupling to bottom pairs can be strongly suppressed. For large $\tan \beta$ this happens for a value of $\sigma(g g \rightarrow h) / S M$ of around 1.4. These models have $110 \mathrm{GeV} \lesssim m_{h} \lesssim 150 \mathrm{GeV}$, where the decays into $b \bar{b}$ of the SM Higgs are important. The suppression in $g_{h b \bar{b}}^{2}$ can also be observed at low $\tan \beta$ (right panel). In this case, the associated values of $m_{h}$ are in the somewhat higher range from $120 \mathrm{GeV}$ to $250 \mathrm{GeV}$, with the strongest suppressions occurring for $m_{h}>150 \mathrm{GeV}$.

The suppression in the coupling to down-type fermions is somewhat reminiscent of the small $\alpha_{\text {eff }}$ scenario [54, $55]$, but there are important differences. In the small $\alpha_{\text {eff }}$ scenario the $g_{h b \bar{b}}$ coupling is suppressed as a result of a cancellation between the tree-level and one-loop contributions. This can happen at large $\tan \beta$, where the radiative effect is enhanced at the same time that the treelevel contribution is somewhat suppressed, thus allowing for a cancellation. Besides large $\tan \beta$, sizable values of $\mu A_{t} / M_{S U S Y}^{2}$ are necessary, and the cancellation is found to happen only for certain values of $m_{A}$ (below or of order $200 \mathrm{GeV}$ ) that are highly correlated with $\tan \beta$ [54]. In contrast, the suppression we find occurs as a result of a cancellation between the tree-level MSSM contribution and those due to the higher-dimension operators (we have checked that the picture remains unchanged by turning off all loop effects). Most importantly, the fact that the suppression occurs at tree-level implies that the couplings to bottom and tau pairs are simultaneously (and strongly) suppressed. This does not tend to happen in the small $\alpha_{\text {eff }}$ scenario, since the radiative enhancements for bottoms and taus happen in different regions of parameter space. Also, in spite of the large number of parameters, there is a clear correlation between the $g_{h b \bar{b}}^{2}$ suppression and the $\sigma(g g \rightarrow h)$ enhancement. The increase in the gluon fusion cross section is due to the destructive interference of the bottom loop in the gluon fusion cross section, and also to light SUSY particles running in the loop.

The suppression of the down-type fermion channels implies a general enhancement in the branching fractions of the remaining channels. The most interesting enhancements are those in the gauge boson channels: $W W, Z Z$ and $\gamma \gamma$. In the left panel of Fig. 9 we show the branching fraction into WW at large $\tan \beta$, again as a function of the gluon fusion production cross section normalized to the SM value, which can be compared to the left panel of Fig. 8. We see that the region where the $h b \bar{b}$ coupling is suppressed is exactly where the WW branching fraction is greatly enhanced, and leads to an interesting Tevatron sensitivity in the $W$ channel over a wide range of $m_{h}$. The left panel of Fig. 9 clearly exhibits how the Tevatron covered (red) points arise. The upper red region corresponds to those models within Tevatron reach in the $h \rightarrow W W$ search, while the two lower red regions contain only points that can be probed in the $h \rightarrow b \bar{b}$ channel. These latter models always have a branching fraction into WW below about $20 \%$.

In the right panel of Fig. 9 we show the $g g \rightarrow h \rightarrow \gamma \gamma$ cross section, normalized to the SM. We see that the di- 
$\tan \beta=20, \mathrm{M}=1 \mathrm{TeV}, \mu=m_{s}=200 \mathrm{GeV}, M_{\mathrm{SUSY}}=300 \mathrm{GeV}, A_{t}=A_{b}=0$

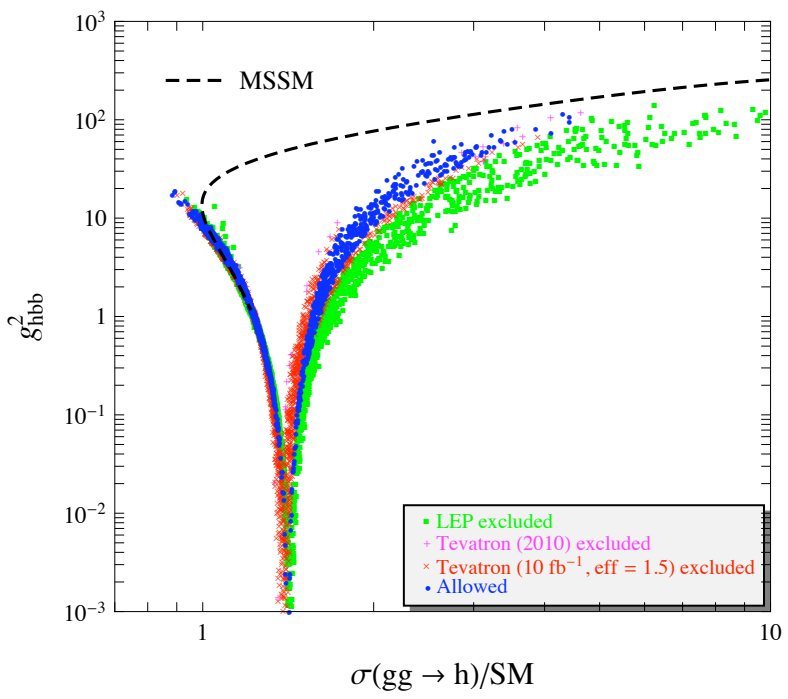

$\tan \beta=2, \mathrm{M}=1 \mathrm{TeV}, \mu=m_{s}=200 \mathrm{GeV}, M_{\mathrm{SUSY}}=300 \mathrm{GeV}, A_{t}=A_{b}=0$

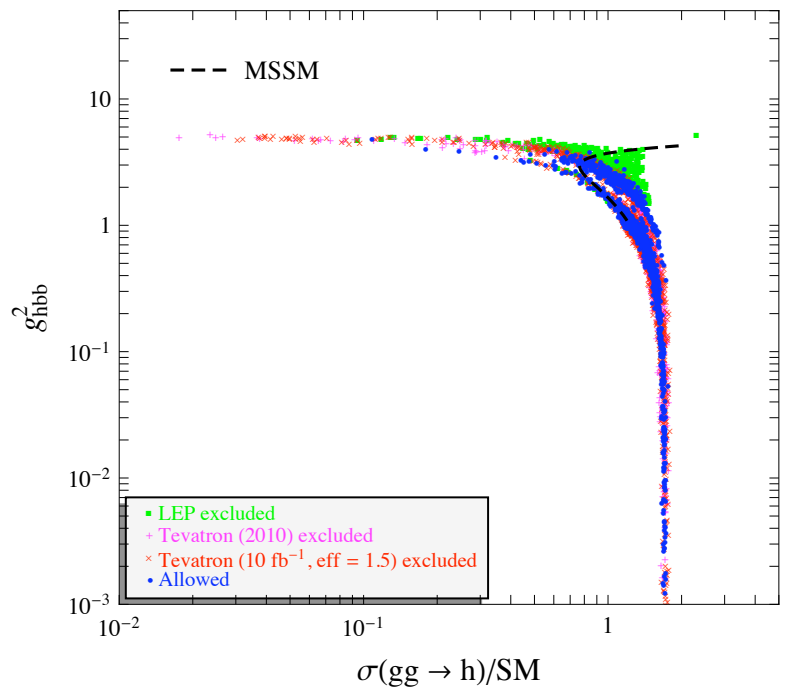

FIG. 8. Effective coupling $g_{h b \bar{b}}^{2}$ for $\tan \beta=20$ (left panel), and for $\tan \beta=2$ (right panel), as a function of the gluon fusion production cross section over the SM value. We show the points excluded by LEP (green), excluded by current Tevatron data (magenta) and the region that will be probed by the Tevatron in the near future (red). The blue points are allowed by all the current and near future experimental constraints. The dashed line corresponds to the MSSM result for the given SUSY spectrum.
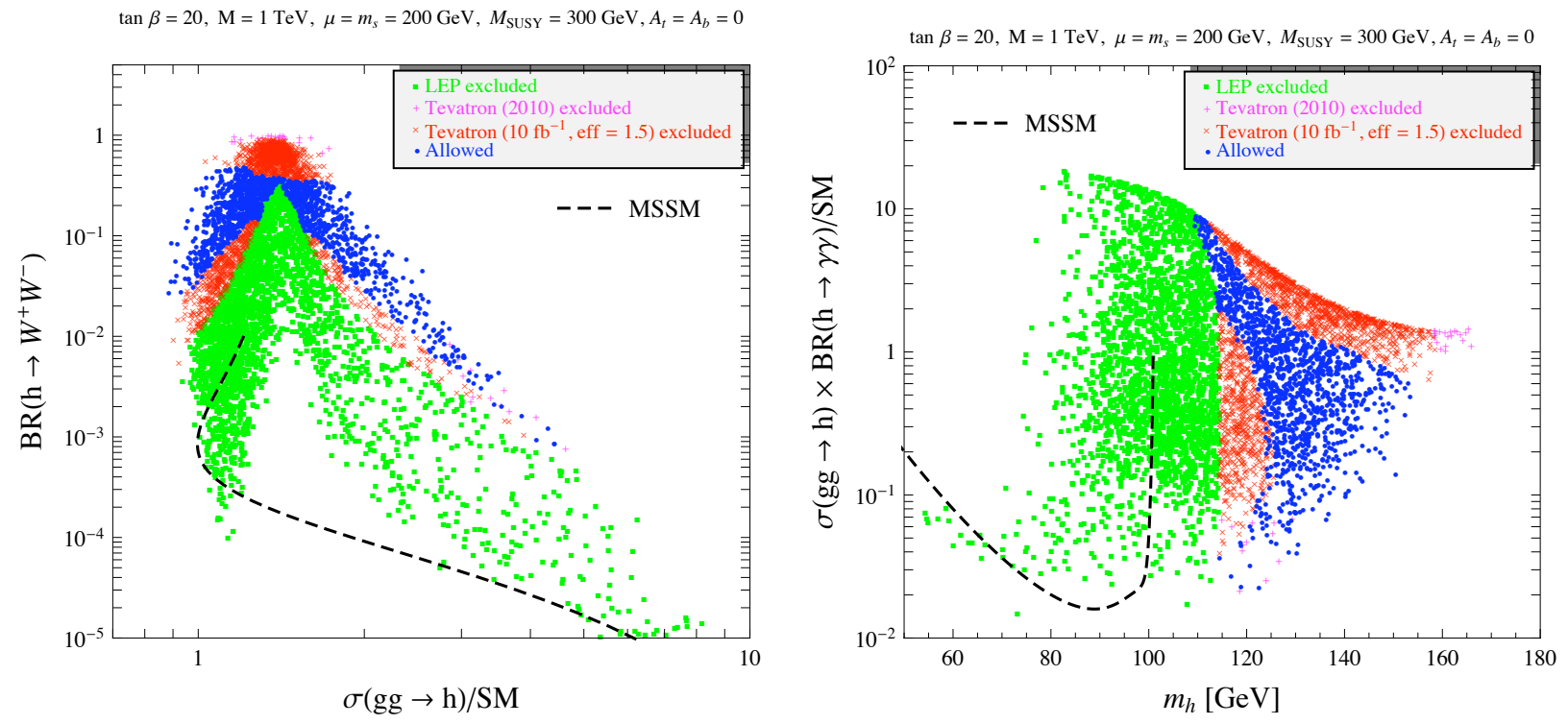

FIG. 9. Left panel: $\mathrm{BR}\left(h \rightarrow W^{+} W^{-}\right)$as a function of the normalized gluon fusion production cross section, for tan $\beta=20$. Right panel: $\sigma(g g \rightarrow h) \times B R(h \rightarrow \gamma \gamma)$, normalized to the $S M$ value, for $\tan \beta=20$. We show the points excluded by LEP (green), excluded by current Tevatron data (magenta) and the region that will be proved by the Tevatron in the near future (red). The blue points are allowed by all the current experimental constraints. The dashed line corresponds to the MSSM result for the given SUSY spectrum.

photon signal can be increased with respect to the SM one by up to a factor of 10 . This strong enhancement is a direct result of the decreased branching fraction into $b \bar{b}$, together with the enhancement in the gluon production cross section discussed above. The points with enhanced signal in the diphoton channel correspond to values of $m_{h}$ between $110 \mathrm{GeV}$ and $130 \mathrm{GeV}$. It is interesting to compare to the latest available diphoton analysis from CDF
[56] and D0 [57]. The CDF analysis, performed with 5.4 $\mathrm{fb}^{-1}$ of data, quotes an observed limit of 18.7-25.9 for the di-photon cross section normalized to the SM. The D0 analysis, with $4.2 \mathrm{fb}^{-1}$, gives a corresponding limit of 11.9-28.3 . [? ] As a result, the enhancement in the di-photon signal we find can be interesting at the Tevatron, and of course it would be spectacular at the LHC. One should also notice that for models with enhanced 
$B R(h \rightarrow b \bar{b})$, the signal into photons can be reduced by up to a factor of 10 .

The $g g \rightarrow h \rightarrow W W$ signal (not shown here) presents the same behavior as the $\gamma \gamma$ one. This can be easily understood as follows. In the SM, the $h \rightarrow \gamma \gamma$ decay mode proceeds via $W$ and top loops, the former giving the dominant effect. In our currently allowed (blue and red) points, the coupling of $h$ to tops and W's is very close to the SM value (the differences are below $2 \%$ ). Although $g_{h b \bar{b}}$ can be enhanced by a factor of 10 , the bottom loop is still a small contribution to the $h \rightarrow \gamma \gamma$ process. Therefore, the partial widths $\Gamma(h \rightarrow W W)$ and $\Gamma(h \rightarrow \gamma \gamma)$ in our model are very close to the SM ones, and the changes in the branching ratios of each channel are common and strictly due to the variation of $B R(h \rightarrow$ $b \bar{b})$ with respect to the SM. Therefore, enhancements in the $W W / Z Z$ channels can also be interestingly large.

With respect to the remaining Higgs bosons, the situation resembles the large $\tan \beta$ regime of the MSSM. Both $H$ and $A$ decay mainly into bottoms and taus, while the charged Higgs goes to either $\tau \nu_{\tau}$ or $t \bar{b}$ depending on its mass. It is also possible for a heavy Higgs to decay into the lightest one: $\mathrm{BR}(H \rightarrow h h)$ can reach $30 \%$, while both $\mathrm{BR}(A \rightarrow h Z)$ and $\mathrm{BR}\left(H^{ \pm} \rightarrow h W^{ \pm}\right)$can reach $10 \%$, provided the decaying Higgs boson mass is above $200 \mathrm{GeV}$. For this mass range, the decay mode into sparticles can also be important, if kinematically allowed.

\section{Large $\tan \beta$ searches: benchmark points}

Having described the main differences of the large tangent beta regime with respect to the MSSM, we show a selected sample of benchmark points.

\section{Scenarios within Tevatron reach}

We start with the points covered in the near future by the Tevatron via the $h \rightarrow b \bar{b}$ search. From Fig. 9 one sees that for these points (lowest red region in the left panel), the signal into $\gamma \gamma$ (and $W W$ ) can be enhanced by at most a factor of 2 . Since such enhancement factors can also be obtained within the MSSM (for instance with sparticle masses around $500 \mathrm{GeV}$ ), we will not show a benchmark point here, but will briefly comment on the main characteristics of these type of models. The Tevatron could claim a hint in the $b \bar{b}$ channel, while at the LHC the signals into $\gamma \gamma$ and $\tau \bar{\tau}$ are enhanced by up to a factor of 2 with respect to the SM, thus allowing for a discovery using these decays modes. Regarding the remaining Higgs bosons, one sees that both $H$ and $A$ decay mainly into bottoms and taus. The gluon fusion production cross section for $H$ and $A$ is around $80 \%$ of the SM-value, while the $b b h$ production becomes an important mechanism due to the large $\tan \beta$ enhancement (the CP-even $H$ has highly suppressed couplings to W's and Z's). Thus a discovery in the $H / A \tau \bar{\tau}$ search may be

\section{POINT G}

\begin{tabular}{|c|c|c|c|}
\hline$m_{A}(\mathrm{GeV})$ & $m_{h}(\mathrm{GeV})$ & $m_{H}(\mathrm{GeV})$ & $m_{H^{ \pm}}(\mathrm{GeV})$ \\
\hline 267 & 148.6 & 297 & 283 \\
\hline$g_{h W W}^{2}$ & $g_{H W W}^{2}$ & $g_{h g g}^{2}$ & $g_{H g g}^{2}$ \\
\hline 0.97 & 0.03 & 1.64 & 0.14 \\
\hline channel & BMSSM (SM) & channel & BMSSM (SM) \\
\hline$h \rightarrow b \bar{b}$ & $0.43(0.20)$ & $h \rightarrow \tau \bar{\tau}$ & $0.07(0.02)$ \\
$h \rightarrow Z Z$ & $0.08(0.05)$ & $h \rightarrow W W$ & $0.41(0.66)$ \\
$H \rightarrow b \bar{b}$ & 0.75 & $H \rightarrow \tau \bar{\tau}$ & 0.13 \\
$A \rightarrow b \bar{b}$ & 0.84 & $A \rightarrow \tau \bar{\tau}$ & 0.14 \\
$H^{ \pm} \rightarrow \tau \nu_{\tau}$ & 0.21 & $H^{ \pm} \rightarrow t \bar{b}$ & 0.75 \\
\hline \multicolumn{4}{|r}{} \\
\hline
\end{tabular}

TABLE VII. Masses and branching fractions in the BMSSM (and in the $S M$ for $h$ ) for point $G$.

feasible [52]. We note also that $A$ and $H$ can be very close in mass, so that the two states cannot be disentangled at the LHC, but rather the signals have to be added up. Because of a sizable branching fraction into the $\tau \nu_{\tau}$ channel, the charged Higgs can be within LHC reach, even for $m_{H^{ \pm}}>m_{t}$ [53]. The way to distinguish such a situation from the MSSM will be through the observation of relatively light superparticles.

As we mentioned before, an interesting possibility is to have sizable branching fractions for the decay modes $H \rightarrow h h, A \rightarrow h Z$ and $H^{ \pm} \rightarrow h W^{ \pm}$. This requires heavy Higgs bosons with a mass above $250 \mathrm{GeV}$. Depending on the details of the SUSY spectrum, also decays into sparticles may be open. We have found that the branching fractions in these multi-Higgs channels are below $10 \%$ in most cases, and that one would still have both $A$ and $H$ decaying sizably into down-type fermions, with $H^{+}$decaying preferably into $t \bar{b}$ but with a non-negligible branching fraction into $\tau \nu_{\tau}$ due to the large $\tan \beta$ enhancement. Provided that $m_{H}>300 \mathrm{GeV}$, the $H \rightarrow h h$ branching fraction can reach values of up to $20-30 \%$, which is interesting since it allows for the potential observation of several Higgs states.

\section{Point G: SM-like Higgs heavier than the MSSM upper bound}

We turn now to the models covered at the Tevatron via the $h \rightarrow W W$ search (upper red region in Figs. 9). We show in Table VII [?] an example where $m_{h}$ is above the maximum attainable value in the $m_{h}$ max scenario of the MSSM, with sparticles at the TeV scale. The $1 / M^{2}$ operators contribute about $45 \mathrm{GeV}$ to $m_{h}$. We notice that in this point the enhancement in the gluon fusion production cross section $\left[1.64 \times \sigma_{S M}(g g \rightarrow h)\right]$ is compensated by the reduction in the $W W$ branching fraction with respect to the SM $(0.41 / 0.66)$, thus resulting in a $g g \rightarrow h \rightarrow W W$ signal close to the $\mathrm{SM}$ one. As can be seen in the right panel of Fig. 9 (interpreted for the $W W$ channel) this is a general feature of the Tevatron covered points in the higher range of $m_{h}$. The nonstandard neutral Higgs bosons, $H$ and $A$, can be detected in the $\tau \bar{\tau}$ (or $\mu \bar{\mu}$ ) channels, as is well known for the large 
POINT H

\begin{tabular}{|c|c|c|c|}
\hline$m_{A}(\mathrm{GeV})$ & $m_{h}(\mathrm{GeV})$ & $m_{H}(\mathrm{GeV})$ & $m_{H^{ \pm}}(\mathrm{GeV})$ \\
\hline 210 & 111.3 & 215 & 225 \\
\hline$g_{h W W}^{2}$ & $g_{H W W}^{2}$ & $g_{h g g}^{2}$ & $g_{H g g}^{2}$ \\
\hline 0.98 & 0.02 & 1.39 & 0.84 \\
\hline channel & BMSSM (SM) & channel & BMSSM (SM) \\
\hline$h \rightarrow b \bar{b}$ & $0.03(0.79)$ & $h \rightarrow \gamma \gamma / 10^{-3}$ & $12.1(2.1)$ \\
$h \rightarrow$ jets & $0.56(0.07)$ & $h \rightarrow W W$ & $0.36(0.05)$ \\
$H \rightarrow b \bar{b}$ & 0.86 & $H \rightarrow \tau \bar{\tau}$ & 0.14 \\
$A \rightarrow b \bar{b}$ & 0.86 & $A \rightarrow \tau \bar{\tau}$ & 0.14 \\
$H^{ \pm} \rightarrow \tau \nu_{\tau}$ & 0.35 & $H^{ \pm} \rightarrow t \bar{b}$ & 0.64 \\
\hline
\end{tabular}

TABLE VIII. Masses and branching fractions in the BMSSM (and in the $S M$ for $h$ ) for point $H$.

$\tan \beta$ region of the MSSM. The charged Higgs can be searched for in the $\tau \nu_{\tau}$ channel. However, we emphasize again that the observation of light SUSY signals would give compelling evidence for BMSSM physics. There are also Tevatron covered (red) points at smaller $m_{h}$ values, around $110 \mathrm{GeV}$ with an enhanced di-photon signal. We discuss these type of scenarios in the next section, together with the Tevatron uncovered (blue points) in the same region.

\section{E. LHC searches}

Referring to Fig. 9 we split the Tevatron uncovered (blue) points according to whether their signal into photons (and W's) is enhanced or suppressed. For the latter case, one has that $h$ decays mainly into bottom and tau pairs. In these scenarios, $h$ can be within the reach of the LHC in the $\tau \bar{\tau}$ channel, and if the suppression of the $\mathrm{ZZ}$ coupling is not extreme, maybe also in the $Z Z \rightarrow 4 l$ channel. Higgs decay chains, such as $H \rightarrow h h \rightarrow b \bar{b} \tau \bar{\tau}$, can also give rise to interesting (if challenging) signatures. We do not show a benchmark point here since the branching fractions of the relevant Higgs decay chain modes will depend on the details of the sparticle spectrum.

Point H: SM-like Higgs with enhanced di-photon signal

We illustrate the features of models with a strong enhancement of the di-photon signal with point $H[?]$ (shown in Table VIII). We see that $h$ is rather light (the $1 / M^{2}$ operators contribute about $10 \mathrm{GeV}$ to $m_{h}$ ), but escaped detection at LEP due to the strong suppression of the $b \bar{b}$ channel. The $g g \rightarrow h \rightarrow \gamma \gamma$ signal is larger than the SM one by a factor of 8 , thus allowing for a very nice and clean detection of $h$ at the LHC. As was discussed in the context of Fig. 9, the same enhacement also occurs for the $W W$ and $Z Z$ channels. Therefore, and in spite of such a light Higgs mass, the $g g \rightarrow h \rightarrow Z Z \rightarrow 4 l$ channel would be at the reach of the LHC.

For the remaining neutral Higgs bosons $(H$ and $A)$, one will have to consider the $\tau \bar{\tau}$ search. The charged Higgs may be detected at the LHC in the $\tau \nu_{\tau}$ channel.
Note that the benchmark point $\mathrm{H}$ has nonstandard Higgs bosons that are too light to allow decays into $h h$. However, given that $h$ is rather light in the region with suppressed $b \bar{b}$ couplings, it is possible that such exotic channels might be open, while still having an interesting di-photon signal. As mentioned before, in such cases it is possible that other channels involving SUSY particles are also open.

\section{CONCLUSIONS}

We studied the Higgs collider phenomenology of BMSSM scenarios, i.e. supersymmetric extensions of the MSSM within an EFT framework where the effects of the BMSSM degrees of freedom enter through higherdimension operators. As emphasized in [8] the first two orders in the $1 / M$ expansion can be phenomenologically significant, and should be included. In the present work, we have performed a model-independent study to highlight the variety of collider signals that become available in such scenarios.

The coupling of the lightest CP-even Higgs to bottom pairs can be suppressed due to cancellations between the MSSM contribution and those from the higher-dimension operators. It does not seem to require a special tuning of parameters and occurs in both the low and large $\tan \beta$ regimes. As a result, the signals in clean channels, such as the di-photon or $W W$ ones, can be greatly enhanced. This suppression in the $h b \bar{b}$ induces an enhancement in the gluon fusion production cross section, beyond the one arising from light sparticles in the loop.

To emphasize the interplay between the Tevatron and the LHC, we have analyzed projections for the Tevatron assuming a total integrated luminosity of $10 \mathrm{fb}^{-1}$ per experiment and a $50 \%$ efficiency improvement in the $W W$ and $b \bar{b}$ search channels with respect to present results. We find that the current Tevatron data already probes a large class of SUSY models, especially in the $W W$ channel. The future projections indicate that the $b \bar{b}$ channel can become effective for a SM-like Higgs search. Moreover, a combination of the $b \bar{b}$ and $W W$ search channels, together with the $\tau \bar{\tau}$ decay mode in the large $\tan \beta$ region, would further enlarge the set of BMSSM models that can be probed at the Tevatron. However, our main interest in this work was to survey the types of signals that might be expected in SUSY scenarios, many of which are not realized in the MSSM limit. Improving the analysis by combining channels and/or moderately increasing the luminosity will not significantly change our conclusions. Lightest CP-even Higgs bosons with masses above $180 \mathrm{GeV}$, that can not be probed by the Tevatron, will be at the reach of the LHC.

Most of the changes in the expected Higgs signals, compared to the MSSM, can be understood in large part from the altered Higgs spectrum. We have surveyed a wide range of possibilities by scanning over the parameter space of the higher-dimension operators. Motivated 
by naturalness arguments, we have chosen the SUSYbreaking scale close to the EW scale, with the BMSSM physics at the $\mathrm{TeV}$ scale. In this case, the contributions from the SUSY particles to the Higgs spectrum are subleading compared to the ones coming from the BMSSM physics. In the case of a heavier SUSY spectrum, and for a scale $M$ such that the effective field theory approach remains valid, the qualitative features of the Higgs phenomenology triggered by the BMSSM physics will be similar. However, a detailed study should be performed for each specific choice of the heavy scale $M$, the scale of SUSY-breaking $m_{S}$, and the $\mu$-term to address the quantitative features of the Higgs sector.

We have defined a number of "benchmark points" in order to discuss the correlations between different Higgs signals. Interestingly, we find that there can be significant mixing in the CP-even Higgs sector, allowing nonnegligible couplings of both CP-even Higgs eigenstates to the EW gauge bosons. In addition, they can both be in the right mass range to decay predominantly into $W$ 's or $Z$ 's, thus enabling a detailed and direct study of the physics of EWSB. For these benchmark points, the $1 / M^{2}$ effects add a few tens of GeV to $m_{h}$, and have a rather relevant impact on the collider phenomenology (but we remind the reader that the $1 / M^{2}$ operators can easily give a much larger contribution to $m_{h}$; see Fig. 1 of Ref. [8]). Furthermore, we have found viable examples where the nonstandard CP-odd Higgs can be produced in charged Higgs decays. Moreover, unusual decay chains such as $h \rightarrow A A$ or $H \rightarrow A A$ are also possible, without $A$ being ultralight. These channels are most interesting in the low $\tan \beta$ region where the $\tan \beta$-enhanced production of the nonstandard Higgs bosons is not available. These Higgs decay chains open the possibility of fully reconstructing the Higgs content of a $2 \mathrm{HDM}$ in such supersymmetric scenarios. We also find scenarios where observing the Higgs sector is more challenging, and would require dedicated studies that go beyond the scope of this work.

In conclusion, we find that Higgs signals in supersymmetric scenarios can be markedly different from those in the MSSM paradigm. If all third generation squarks turn out to be light $\left(m_{S} \leq 300 \mathrm{GeV}\right)$, given the LEP Higgs mass bounds, this will imply a clear case for BMSSM physics. The heavier degrees of freedom could be at the kinematic reach of the LHC, but depending on their nature the direct discovery might be elusive. In either case, supersymmetric Higgs searches can provide evidence of physics beyond the MSSM.

\section{ACKNOWLEDGMENTS}

We would like to thank Oliver Brein and Karina Williams for making an unofficial version of the HiggsBounds code available to us, and for the help provided. J.Z would like to thank the Theory Division of Fermilab for hospitality during the final stages of this work. Fermilab is operated by the Fermi Research Alliance, LLC under Contract No. DE-AC02-07CH11359 with the U.S. Department of Energy. E.P. is supported by DOE Grant No. DE-FG02-92ER40699. The work of J.Z is supported by the Swiss National Science Foundation (SNF) under Contract No. 200020-126691.
[1] A. Strumia, Phys. Lett. B 466, 107 (1999) [arXiv:hep$\mathrm{ph} / 9906266]$.

[2] A. Brignole, J. A. Casas, J. R. Espinosa and I. Navarro, Nucl. Phys. B 666, 105 (2003) [arXiv:hep-ph/0301121].

[3] M. Dine, N. Seiberg and S. Thomas, Phys. Rev. D 76, 095004 (2007) [arXiv:0707.0005 [hep-ph]].

[4] I. Antoniadis, E. Dudas and D. M. Ghilencea, JHEP 0803, 045 (2008) [arXiv:0708.0383 [hep-th]]. I. Antoniadis, E. Dudas, D. M. Ghilencea and P. Tziveloglou, Nucl. Phys. B 808, 155 (2009) [arXiv:0806.3778 [hepph]]. I. Antoniadis, E. Dudas, D. M. Ghilencea and P. Tziveloglou, AIP Conf. Proc. 1078, 175 (2009) [arXiv:0809.4598 [hep-ph]].

[5] I. Antoniadis, E. Dudas, D. M. Ghilencea and P. Tziveloglou, Nucl. Phys. B 831, 133 (2010) [arXiv:0910.1100 [hep-ph]].

[6] L. Randall, JHEP 0802, 084 (2008)

[7] P. Batra and E. Pontón, Phys. Rev. D 79, 035001 (2009) [arXiv:0809.3453 [hep-ph]].

For earlier references see P. Fayet, Nucl. Phys. B 90, 104 (1975), R. K. Kaul and P. Majumdar, Nucl. Phys. B 199, 36 (1982), and also H. E. Haber and G. L. Kane, Phys. Rept. 117, 75 (1985).

[8] M. Carena, K. Kong, E. Pontón and J. Zurita, Phys. Rev.
D 81, 015001 (2010) [arXiv:0909.5434 [hep-ph]].

[9] K. Blum, C. Delaunay and Y. Hochberg, Phys. Rev. D 80, 075004 (2009) [arXiv:0905.1701 [hep-ph]].

[10] J. A. Casas, J. R. Espinosa and I. Hidalgo, JHEP 0401, 008 (2004) [arXiv:hep-ph/0310137].

[11] S. Cassel, D. M. Ghilencea and G. G. Ross, Nucl. Phys. B 825, 203 (2010) [arXiv:0903.1115 [hep-ph]].

[12] K. Cheung, S. Y. Choi and J. Song, Phys. Lett. B 677, 54 (2009) [arXiv:0903.3175 [hep-ph]].

[13] M. Berg, J. Edsjo, P. Gondolo, E. Lundstrom and S. Sjors, JCAP 0908, 035 (2009) [arXiv:0906.0583 [hep$\mathrm{ph}]$.

[14] N. Bernal and A. Goudelis, JCAP 1003, 007 (2010) [arXiv:0912.3905 [hep-ph]].

[15] N. Bernal, K. Blum, M. Losada and Y. Nir, JHEP 0908, 053 (2009) [arXiv:0906.4696 [hep-ph]].

[16] C. Grojean, G. Servant and J. D. Wells, Phys. Rev. D 71, 036001 (2005) [arXiv:hep-ph/0407019].

[17] D. Bodeker, L. Fromme, S. J. Huber and M. Seniuch, JHEP 0502, 026 (2005) [arXiv:hep-ph/0412366].

[18] C. Delaunay, C. Grojean and J. D. Wells, JHEP 0804, 029 (2008) [arXiv:0711.2511 [hep-ph]].

[19] A. Noble and M. Perelstein, Phys. Rev. D 78, 063518 (2008) [arXiv:0711.3018 [hep-ph]]. 
[20] K. Blum and Y. Nir, Phys. Rev. D 78, 035005 (2008) [arXiv:0805.0097 [hep-ph]]. K. Blum, C. Delaunay, M. Losada, Y. Nir and S. Tulin, arXiv:1003.2447 [hep-ph].

[21] M. E. Peskin and T. Takeuchi, Phys. Rev. Lett. 65, 964 (1990); Phys. Rev. D 46, 381 (1992).

[22] P. Bechtle, O. Brein, S. Heinemeyer, G. Weiglein and K. E. Williams, arXiv:0811.4169 [hep-ph].

[23] P. Bechtle, O. Brein, S. Heinemeyer, G. Weiglein and K. E. Williams, arXiv:0905.2190 [hep-ph].

[24] [LEP Higgs Working Group for Higgs boson searches and ALEPH Collaboration and DELPHI Collaboration and L3 Collaboration and OPAL Collaboration], arXiv:hepex/0107031.

[25] T. Aaltonen et al. [CDF and D0 Collaborations], arXiv:1001.4162 [hep-ex].

[26] http://www-d0.fnal.gov/Run2Physics/WWW/results/ prelim/HIGGS/H87/H87.pdf

[27] A. Djouadi, J. Kalinowski and M. Spira, Comput. Phys. Commun. 108, 56 (1998) [arXiv:hep-ph/9704448].

[28] M. Spira, Fortsch. Phys. 46, 203 (1998) [arXiv:hep$\mathrm{ph} / 9705337]$.

[29] M. S. Carena, J. R. Espinosa, M. Quiros and C. E. M. Wagner, Phys. Lett. B 355, 209 (1995) [arXiv:hep-ph/9504316].

[30] M. S. Carena, M. Olechowski, S. Pokorski and C. E. M. Wagner, Nucl. Phys. B 426, 269 (1994) [arXiv:hep-ph/9402253]. D. M. Pierce, J. A. Bagger, K. T. Matchev and R. j. Zhang, Nucl. Phys. B 491, 3 (1997) [arXiv:hep-ph/9606211]. L. J. Hall, R. Rattazzi and U. Sarid, Phys. Rev. D 50, 7048 (1994) [arXiv:hep$\mathrm{ph} / 9306309]$.

[31] M. S. Carena, D. Garcia, U. Nierste and C. E. M. Wagner, Nucl. Phys. B 577, 88 (2000) [arXiv:hepph/9912516]. J. Guasch, P. Hafliger and M. Spira, Phys. Rev. D 68, 115001 (2003) [arXiv:hep-ph/0305101].

[32] S. Dawson, A. Djouadi and M. Spira, Phys. Rev. Lett. 77, 16 (1996) [arXiv:hep-ph/9603423].

[33] V. M. Abazov et al. [D0 Collaboration], Phys. Lett. B 660, 449 (2008) [arXiv:0712.3805 [hep-ex]].

[34] T. Aaltonen et al. [CDF Collaboration], Phys. Rev. Lett. 102, 121801 (2009) [arXiv:0811.2512 [hep-ex]].

[35] M. S. Carena, R. J. Hernández and A. Menon, in preparation.

[36] See the talk by Wade Fisher at the "Rencontres de Moriond, 2010".

[37] P. Draper, T. Liu and C. E. M. Wagner, Phys. Rev. D 80, 035025 (2009) [arXiv:0905.4721 [hep-ph]].
[38] [LEP Higgs Working Group for Higgs boson searches and ALEPH Collaboration and DELPHI Collaboration and L3 Collaboration and OPAL Collaboration], arXiv:hepex/0107030.

[39] S. Schael et al. [ALEPH Collaboration], Eur. Phys. J. C 47, 547 (2006) [arXiv:hep-ex/0602042].

[40] H. M. Georgi, S. L. Glashow, M. E. Machacek and D. V. Nanopoulos, Phys. Rev. Lett. 40, 692 (1978).

[41] M. Spira, A. Djouadi, D. Graudenz and P. M. Zerwas, Nucl. Phys. B 453, 17 (1995) [arXiv:hep-ph/9504378].

[42] M. Spira, arXiv:hep-ph/9510347.

[43] R. V. Harlander and W. B. Kilgore, Phys. Rev. Lett. 88, 201801 (2002) [arXiv:hep-ph/0201206].

[44] C. Anastasiou and K. Melnikov, Nucl. Phys. B 646, 220 (2002) [arXiv:hep-ph/0207004].

[45] V. Ravindran, J. Smith and W. L. van Neerven, Nucl. Phys. B 665, 325 (2003) [arXiv:hep-ph/0302135].

[46] S. Catani, D. de Florian, M. Grazzini and P. Nason, JHEP 0307, 028 (2003) [arXiv:hep-ph/0306211].

[47] C. Anastasiou, R. Boughezal and F. Petriello, JHEP 0904, 003 (2009) [arXiv:0811.3458 [hep-ph]].

[48] D. de Florian and M. Grazzini, Phys. Lett. B 674, 291 (2009) [arXiv:0901.2427 [hep-ph]].

[49] M. Carena, T. Han, G. Y. Huang and C. E. M. Wagner, JHEP 0804, 092 (2008) [arXiv:0712.2466 [hep-ph]].

[50] R. Dermisek and J. F. Gunion, Phys. Rev. D 79, 055014 (2009) [arXiv:0811.3537 [hep-ph]]. R. Dermisek and J. F. Gunion, Phys. Rev. D 81, 055001 (2010) [arXiv:0911.2460 [hep-ph]]. R. Dermisek and J. F. Gunion, arXiv:1002.1971 [hep-ph].

[51] K. Cheung, J. Song and Q. S. Yan, Phys. Rev. Lett. 99, 031801 (2007) [arXiv:hep-ph/0703149].

[52] ATLAS Detector and Physics Peformance TDR. CERN, 1999.

[53] The ATLAS Collaboration, G. Aad et. al., Expected Performance of the ATLAS Experiment - Detector, Trigger and Physics, http://xxx.lanl.gov/abs/0901.0512.

[54] M. S. Carena, S. Heinemeyer, C. E. M. Wagner and G. Weiglein, Eur. Phys. J. C 26, 601 (2003) [arXiv:hep$\mathrm{ph} / 0202167]$.

[55] M. S. Carena, S. Heinemeyer, C. E. M. Wagner and G. Weiglein, Eur. Phys. J. C 45, 797 (2006) [arXiv:hep$\mathrm{ph} / 0511023]$.

[56] http://www-cdf.fnal.gov/physics/new/hdg/ Results_files/results/hgamgam_jan10_cdf10065_ HiggsGamGam54Public.pdf

[57] http://www-d0.fnal.gov/Run2Physics/WWW/results/ prelim/HIGGS/H66/H66.pdf 